\title{
Geminate Recombination in the Presence of Scavengers: The New Vision of the Old Problem
}

\author{
S. G. Fedorenko, ${ }^{1}$ A. A. Kipriyanov, ${ }^{1,2}$ and A. B. Doktorov ${ }^{1,2}$ \\ ${ }^{1}$ Institute of Chemical Kinetics and Combustion, SB RAS, 630090 Novosibirsk, Russia \\ ${ }^{2}$ Department of General Physics, Novosibirsk State University, 630090 Novosibirsk, Russia
}

Correspondence should be addressed to S. G. Fedorenko, fedorenk@kinetics.nsc.ru

Received 6 September 2010; Accepted 25 January 2011

Academic Editor: Ali Hussain Reshak

Copyright ( $) 2011$ S. G. Fedorenko et al. This is an open access article distributed under the Creative Commons Attribution License, which permits unrestricted use, distribution, and reproduction in any medium, provided the original work is properly cited.

A geminate reaction between $A$ and $B$ reactants affected by the bulk reaction between $B$ and scavengers $C$ is discussed. The exact solution of the problem obtained recently for randomly walking $B$ reactant (excess electron) is compared with the superposition approximation commonly used to interpret experimental data. Distinctions related to the important role of time correlations between geminate and bulk reactions are analyzed. The largest deviations exponentially growing in time are observed for geminate reaction rate in the presence of scavengers. It is shown that superposition approximation can decrease essentially the ultimate probability of geminate recombination. The difference is great enough to lead to qualitatively incorrect description of the experiment even at small concentration of scavengers. This, in turn, may give rise to considerable errors in the determination of geminate pair parameters or, alternatively, to the wrong information about bulk kinetics of electron scavenging.

\section{Introduction}

More than fifty years ago Monchick [1] formulated the problem of the influence of scavengers with a uniform concentration $[C]$ in the bulk on the evolution of a geminate pair formed by photo or pulse radiation in solution. This problem subsequently called a "scavenger problem" [211] in the most simple case may be described as follows. After thermalization in chemically inert solvent, $A$ and $B$ radicals or ion-radicals produced from a "parent" molecule form geminate (spatially correlated) pairs [12-17]. The subsequent reaction

$$
A+B \longrightarrow P
$$

is divided into two stages: geminate and bulk recombinations. Geminate reaction proceeds between spatially correlated radicals formed from one and the same parent molecule. As a rule, this takes several nanoseconds [18, 19]. Bulk reaction is recombination of radicals that left initial geminate pairs to find themselves in the bulk. At small concentration of such pairs this stage differs essentially from a geminate one in the time scale and is commonly observed on the times from micro- to milliseconds. Ignoring this stage enables one to treat reaction (1) as recombination of isolated geminate pairs [20-22].

Under the above conditions, the simplest bulk reaction competing with geminate one is possible on addition of uniformly distributed $C$ acceptors of one (say $B$ ) type of radicals to the solution. Usually $C$ reactants are in excess (as compared to $B s$ ), thus their concentration remains almost unchanged

$$
B+C \longrightarrow C+D
$$

Note that the excess of $C$ reactants is no barrier to taking into account solely pair encounters of $B$ with $C$ reactants in considering small concentrations of scavengers $[C]$. The problem consists in calculating the decay kinetics of $A$ or $B$ reactants (or the formation kinetics of $P$ or $D$ products of geminate (1) or bulk (2) reactions, resp.), and the dependence of $P$ or $D$ product yield on $C$ acceptors concentration in such a two-stage reaction.

In his first work Monchick [1] allowed for the influence of scavengers by introducing extraterm proportional to 
constant decay rate on acceptors $[C] k$ into the Smoluchowski equation defining the evolution of isolated geminate pair:

$$
\begin{aligned}
\partial_{t} G\left(\vec{r}, t \mid \vec{r}^{\prime}, 0\right)= & D_{A B} \nabla_{\vec{r}}\left[\nabla_{\vec{r}} G+\frac{G}{k_{B} T} \nabla_{\vec{r}} \Phi\right] \\
& -[C] k G,
\end{aligned}
$$

where $D_{A B}=D_{A}+D_{B}$ is the mutual diffusion coefficient and $\Phi$ is a potential of mean force between two radicals. Geminate recombination is defined by partially absorbing boundary condition at the contact radius, while $k$ is a bimolecular rate constant of bulk reaction (2) depending on mutual mobility of $B$ and $C$ reactants. Physically such a simple description of the scavenging effect has been justified by the assumption that diffusion profile of a geminate pair is gradually reduced in time, but spatial distribution is not affected by the presence of scavengers. Simple transformation

$$
G\left(\vec{r}, t \mid \vec{r}^{\prime}, 0\right)=\exp (-[C] k t) G_{A B}\left(\vec{r}, t \mid \vec{r}^{\prime}, 0\right)
$$

converts (3) to the Smoluchowski equation in the absence of scavengers for $G_{A B}$. In fact, this means that reactions (1) and (2) are considered to be independent of one another. This assumption of the independent course of geminate and bulk reactions is the basic postulate of conventional phenomenological theory developed further in a number of papers [2-10]. Based on this assumption, experimentally important quantity, total scavenging probability $\mathcal{P}_{D}(t \rightarrow$ $\infty$ ) (that is defined as the probability that $B$ radical react with a scavenger before recombination with $A$ radical), can be related to the survival probability of a geminate pair as follows:

$$
\mathcal{P}_{D}(t \longrightarrow \infty) \simeq[C] k \int_{0}^{\infty} d t \Omega(t) e^{-[C] k t} .
$$

This equation shows that in phenomenological theory the total scavenging probability is proportional to the Laplace transform of the geminate pair survival probability in the absence of scavengers $\Omega(t)$. Relation (4) has become a theoretical basis for the development of powerful experimental method for the investigation of geminate recombination kinetics by concentration dependence measurements of total scavenging yield. The devised method, along with the method of external electric field, has become a very important tool for studying the kinetics of ion-radical processes. Its extensive applications is related to the discovery of short-lived particles, in particular, excess electron. This method described in detail in textbooks and reviews [21, 2326], in fact, compares with the method of investigating quantum yield of luminescence concentration quenching both in ideology and significance. Despite recent progress of the new methods of direct observation of geminate kinetics and excess electron capture scavenger method, as a universal way of affecting geminate recombination by bulk reactants, is still of importance. It has found application in identification of kinetic mechanism of multistage bulk-geminate reactions in various systems [27-30].

At the same time, only one attempt has been made in the literature to substantiate the phenomenological theory based on relations (3)-(5). In paper [11] the phenomenological theory (corrected for nonstationary course of bulk reaction) was confirmed by a more consistent many-particle theory based on superposition decoupling of three-particle correlation in the reduced distribution function at small concentrations of acceptors. However, even in the binary approach the use of superposition decoupling in manyparticle derivation of kinetic equations for some reactions proved to be incorrect and was criticized in a number of works [31-33]. Recently the exactly solvable many-particle model has been proposed for the "scavenger problem" in order to take into account time correlations between bulk and geminate reactions for the first time [34]. This model allows us to obtain exact kinetic equations for the general type of $A$ and $C$ reactants mobility and the Coulomb interaction in a geminate pair [35]. The most essential assumption of the model is that $B$ particle of a geminate pair (excess electron) reacting with scavengers $C$ moves by infinitely large jumps (the hopping mechanism [36-42]). The hopping mechanism has been used more than once to describe the scavenging of an excess electron moving by random walks in solutions [40-44]. This assumption of the large jumps is justified by the well-known fact that mean free path of excess electrons is fairly large in some solvent of low polarity $[23,43,45](\approx 60-200 \AA)$. In Letters $[46,47]$ two simple microscopic models of reactions (1) and (2) admitting analytical solution of exactly solvable model equations [34] were examined. It has been shown that the role of time correlations in the course of reactions (1) and (2) may be significant even at small concentration of scavengers $[C]$. The analysis of relative deviations between the exact solution of the "scavenger problem" and superposition approximation shows that superposition approximation substantially underestimates the rate of geminate recombination and the yield of geminate reaction product in the presence of scavengers. Thus it is very important to investigate the parameters value areas where correlations are so significant that approximate theories become unsuitable for interpretation of experimental evidence.

The outline of the paper is as follows: the next section presents conventional theory and superposition approximation. In Section 3 two simple microscopic models of the reacting system are described, and calculations of experimentally measured quantities by the recipes of the above approximate theories are performed. In the first model chosen (reacting "black" spheres, the hopping motion of $B$ and $C$ reactants) the bulk reaction kinetics develop exponentially in time, thus the results of both approximate theories become kinetically identical. We ignore deliberately nonstationarity effects responsible for additional correlations in the system, and focus our attention on the simplest case (most favourable for approximate theories) where $B$ reactant decays at a constant rate on acceptors. The second microscopic model refers to a more realistic case of diffusion motion of scavengers in liquid solutions, and, therefore, contains additional correlations related to the presence of diffusion nonstationary part of decay kinetics on scavengers. In Section 4 we define the exactly solvable many-particle problem, and give analytical solution for hopping and 
diffusion motion of scavengers. Section 5 presents the detailed analysis of relative deviations of approximate theories from the exactly solvable model in the area of small concentrations of scavengers.

\section{Conventional Theory and Superposition Approximation}

Commonly, $\mathcal{P}_{B}(t)$ (survival probability of $B$ reactant) and $\mathcal{P}_{P}(t)$ (accumulation kinetics of geminate reaction products) are quantities measured experimentally. The survival probability $\mathcal{P}_{A}(t)$ of $A$ reactant, the kinetics of scavenging, $\mathcal{P}_{D}(t)$, can be found by using the normalization conditions $[34,35]$ :

$$
\mathcal{P}_{A}(t)+\mathcal{P}_{P}(t)=1, \quad \mathcal{P}_{B}(t)+\mathcal{P}_{D}(t)+\mathcal{P}_{P}(t)=1 .
$$

The last relation is simplified in the stationary limit $t \rightarrow \infty$, thus the experimentally observed ultimate probability of recombination $\mathcal{P}_{P}(t \rightarrow \infty)$ and total scavenging yield $\mathcal{P}_{D}(t \rightarrow \infty)$ obey the relation:

$$
\mathcal{P}_{D}(t \longrightarrow \infty)+\mathcal{P}_{P}(t \longrightarrow \infty)=1 .
$$

Besides, recently, a new method for the determination of the bulk reaction rate constant has been proposed which is based on direct experimental measurements of the formation rate of geminate recombination products in the presence of bulk scavengers $\dot{\mathcal{P}}_{P}(t)[48]$.

Conventional theory [1-10] enters constant decay rate on acceptors $[C] k$ into microscopic equations of a geminate pair, therefore, the influence of competing bulk reaction is defined only by this parameter that is the product of the acceptor concentration $[C]$ and stationary rate constant $k$ of $B$ particles decay on these acceptors. Thus we have the recipe for the calculation of experimentally measured quantities:

$$
\begin{gathered}
\mathcal{P}_{P}(t) \simeq \mathcal{P}_{P}^{c t}(t) \equiv \int_{0}^{t} d \tau K_{g}(\tau) \exp (-[C] k \tau), \\
\mathcal{P}_{B}(t) \simeq \mathcal{P}_{B}^{c t}(t) \equiv \Omega(t) \exp (-[C] k t) .
\end{gathered}
$$

As usual, geminate reaction rate in the absence of acceptors $K_{g}(t)$ is expressed in terms of the elementary rate $w_{A B}(\vec{r})$ :

$$
K_{g}(t)=\int d \vec{r}^{\prime} \int d \vec{r} w_{A B}(\vec{r}) G_{A B}\left(\vec{r}, t \mid \vec{r}^{\prime}, 0\right) P\left(\vec{r}^{\prime}\right),
$$

where $P\left(\vec{r}^{\prime}\right)$ is the initial distribution of reactants in geminate pairs, and $G_{A B}\left(\vec{r}, t \mid \vec{r}^{\prime}, 0\right)$ is the probability density to find $A$ and $B$ reactants at the relative distance $\vec{r}$ at the instant of time $t$ if at the initial moment $t=0$ they were at the distance $\vec{r}^{\prime}$ (the Green function). It obeys the equation

$$
\partial_{t} G_{A B}\left(\vec{r}, t \mid \vec{r}^{\prime}, 0\right)=\left[\hat{\mathcal{L}}_{A B}-w_{A B}(\vec{r})\right] G_{A B}\left(\vec{r}, t \mid \vec{r}^{\prime}, 0\right)
$$

with the initial condition

$$
G_{A B}\left(\vec{r}, 0 \mid \vec{r}^{\prime}, 0\right)=\delta\left(\vec{r}-\vec{r}^{\prime}\right) .
$$

In (11), $\hat{\mathscr{L}}_{A B}$ is the integral operator defining Markovian random walks of $A$ and $B$ in relative coordinates of the pair $A B$. The force interaction is included.

The survival probability of geminate pairs in the absence of competing bulk reaction is defined by the expression:

$$
\Omega(t)=1-\int_{0}^{t} d \tau K_{g}(\tau)
$$

Superposition approximation [11] adjusts phenomenological theory in view of non-stationarity of the bulk kinetics. As a result, accumulation kinetics of geminate reaction products is obtained by integrating the product of the bulk reaction kinetics and pure geminate reaction rate:

$$
\mathcal{P}_{P}(t) \simeq \mathcal{P}_{P}^{s p}(t) \equiv \int_{0}^{t} d \tau K_{g}(\tau) N(\tau)
$$

while $B$ reactant decay kinetics is expressed as the product of bulk and geminate kinetics:

$$
\mathcal{P}_{B}(t) \simeq \mathcal{P}_{B}^{s p}(t) \equiv N(t) \Omega(t)
$$

Note that superposition approximation actually substantiates the validity of papers $[49,50]$ wherein formulae (14), (15) were introduced intuitively.

For dilute solution of reactants the bulk reaction kinetics may be expressed as $[21,22]$ :

$$
N(t)=\exp \left\{-[C] \int_{0}^{t} d \tau K(\tau)\right\} .
$$

Note that the expression in exponent (16) is linear in concentration of acceptors $[C]$, since it allows solely for binary collisions of reactants in the bulk. In the general case of arbitrary mobilities of $B$ and $C$ reactants, taking account of many-particle collisions results in nonlinear corrections in concentration corresponding to the competition of acceptors with each other. Only in the case of immobile $B$ particle ("target problem" $[51,52])$ nonlinear corrections become zero, and formula (16) gives the exact many-particle description of the bulk reaction.

In the absence of initial correlations between reactants the rate constant of the bulk reaction is

$$
K(t)=\int d \vec{r}^{\prime} \int d \vec{r} w_{B C}(\vec{r}) G_{B C}\left(\vec{r}, t \mid \vec{r}^{\prime}, 0\right) .
$$

Stationary rate constant of the decay on acceptors $k$ appearing in formulae (8) and (9) is determined from (17):

$$
k=\lim _{t \rightarrow \infty} K(t) .
$$

The Green function $G_{B C}\left(\vec{r}, t \mid \vec{r}^{\prime}, 0\right)$ of the pair $B C$ is defined by the equation

$$
\partial_{t} G_{B C}\left(\vec{r}, t \mid \vec{r}^{\prime}, 0\right)=\left[\hat{\mathscr{L}}_{B C}-w_{B C}(\vec{r})\right] G_{B C}\left(\vec{r}, t \mid \vec{r}^{\prime}, 0\right)
$$

with the initial condition of type (12). As in (11), $\hat{\mathcal{L}}_{B C}$ is the integral operator describing translational motion of 
reactants in relative coordinates of the reaction pair, and bulk reaction between $B$ and $C$ reactants is specified by the elementary rate $w_{B C}(\vec{r})$ which depends on their relative position.

Thus in both approximate theories the experimentally measured quantities (8), (9), and (14), (15) are expressed in a simple way in terms of pure geminate and bulk reactions kinetics. This indicates that, in fact, both conventional theory and superposition approximation consider these reactions as proceeding independently.

\section{Microscopic Model of the "Scavenger Problem"}

We believe the above independence of bulk and geminate reactions in the "scavenger problem" to be unjustified at least for two physical reasons. First, on intrusion into a geminate pair, an acceptor is to affect essentially the course of geminate reaction, with the most significant changes being determined by the interruption of recontacts between $A$ and $B$ reactants. Second, the situation of highly mobile $B$ reactant and relatively slow $C$ reactants is possible. In this case the entry of reactants into the bulk results in arresting geminate reaction with the subsequent initiation of the bulk one. In other words, generally speaking, the course of both reactions should be correlated in time.

3.1. Geminate Reaction. For further investigation we choose the simplest microscopic model of the reacting system. We shall describe a geminate reaction by the model of isotropic "black" sphere of the radius $R_{A B}$ [53]. The "black" sphere model excludes recontacts of reactants and, consequently, related time correlations in the system.

Let us take that uncharged $A$ reactant moves by continual diffusion, while the motion of $B$ reactant (excess electron) is described by the model of infinite jumps with the mean frequency $\tau_{B}^{-1}$ (the so-called two-scale migration [54]):

$$
\hat{\mathcal{L}}_{A B}=D_{A} \nabla_{\vec{r}}^{2}-\tau_{B}^{-1} \hat{I}
$$

Here $\hat{I}$ is a unit operator.

Starting with the initial distance $r_{0}$

$$
P(\vec{r})=P(r)=\frac{\delta\left(r-r_{0}\right)}{4 \pi r r_{0}},
$$

we easily obtain that at $r_{0}>R_{A B}$ geminate reaction rate in the absence of bulk acceptors is defined as

$$
\begin{gathered}
K_{g}\left(t, r_{0}\right)=\tilde{K}_{g}\left(t, r_{0}\right) e^{-t / \tau_{B}} \\
\tilde{K}_{g}\left(t, r_{0}\right)=\frac{R_{A B}}{r_{0}} \sqrt{\frac{\tilde{\tau}_{g}}{4 \pi t^{3}}} \exp \left(-\frac{\tilde{\tau}_{g}}{4 t}\right),
\end{gathered}
$$

where $\tilde{\tau}_{g}=\left(r_{0}-R_{A B}\right)^{2} / D_{A}$ is the characteristic decay time of the geminate pair, and $\widetilde{K}_{g}$ is the geminate reaction rate for the case of immobile $B$ reactant. Further, quantities related to the problem with immobile $B$ particle will be marked by a tilde.
By analogy with $\tilde{\tau}_{g}$ we introduce the characteristic decay time of the geminate pair $\tau_{g}$ for the case of two-scale migration defining it as the characteristic decay time of the function $K_{g}\left(t, r_{0}\right)$. Obviously, it is proportional to the time moment $t_{m}$ of the maximum of this function $\tau_{g}=\lambda t_{m}$. The proportionality coefficient $\lambda$ is determined from the coincidence condition of $\tau_{g}$ and $\tilde{\tau}_{g}$ at $\tau_{B} \rightarrow \infty$. It is easily seen that $\lambda=6$. So for $\tau_{g}$,

$$
\tau_{g}=\frac{3}{2} \tau_{B}\left(\sqrt{9+\frac{4 \tilde{\tau}_{g}}{\tau_{B}}}-3\right) \approx 3 \sqrt{\tilde{\tau}_{g} \tau_{B}}, \quad \tau_{B} \ll \tilde{\tau}_{g} .
$$

The use of (22) in (13) with the subsequent integration gives the expression for the survival probability:

$$
\begin{aligned}
\Omega\left(t, r_{0}\right)=1-\frac{R_{A B}}{2 r_{0}}[ & e^{-\sqrt{\eta}} \operatorname{erfc}\left(\sqrt{\frac{\tilde{\tau}_{g}}{4 t}}-\sqrt{\frac{t}{\tau_{B}}}\right) \\
& \left.+e^{\sqrt{\eta}} \operatorname{erfc}\left(\sqrt{\frac{\tilde{\tau}_{g}}{4 t}}+\sqrt{\frac{t}{\tau_{B}}}\right)\right],
\end{aligned}
$$

where the introduced parameter

$$
\eta=\frac{\tilde{\tau}_{g}}{\tau_{B}}=\frac{\left(r_{0}-R_{A B}\right)^{2}}{D_{A} \tau_{B}}
$$

is defined by the ratio of $\tilde{\tau}_{g}$ to $B$ reactant residence time in the pair $\tau_{B}$. Since geminate reaction is arrested immediately after the first jump of $B$ reactant, the depth of the reaction course depends noticeably on the parameter $\eta$ :

$$
\Omega\left(\infty, r_{0}\right) \equiv \Omega_{\infty}\left(r_{0}\right)=1-\frac{R_{A B}}{r_{0}} \exp (-\sqrt{\eta}) .
$$

Only in the limit $\eta \rightarrow 0$ it is equal to the probability of the diffusion entry of reactants into the bulk: $1-R_{A B} / r_{0}$. In this limiting case of immobile $B\left(\tau_{B} \rightarrow \infty\right)$ expression (24) is easily seen to turn into the well-known diffusion result [21]:

$$
\lim _{\tau_{B} \rightarrow \infty} \Omega\left(t, r_{0}\right) \equiv \tilde{\Omega}\left(t, r_{0}\right)=1-\frac{R_{A B}}{r_{0}} \operatorname{erfc}\left(\sqrt{\frac{\tilde{\tau}_{g}}{4 t}}\right) .
$$

Finally, it is worth noting that our microscopic model neglects the Coulomb interaction in a geminate pair and considers the case of the photodetachment process of excess electron generation in solution. However, our results can readily be applied to the description of charged geminate pairs in water-like solutions for which the Onsager radius $\left|r_{c}\right| \leq 10 \AA$. As follows from $[55,56]$, in this case it will suffice to perform the following substitution:

$$
\begin{gathered}
\tilde{\tau}_{g} \longrightarrow \tilde{\tau}_{g}^{c}=\frac{r_{c}^{2}}{4 D_{A}}\left[\operatorname{coth}\left(\frac{r_{c}}{2 r_{0}}\right)-\operatorname{coth}\left(\frac{r_{c}}{2 R_{A B}}\right)\right]^{2}, \\
\frac{R_{A B}}{r_{0}} \longrightarrow \frac{r_{\mathrm{eff}}\left(R_{A B}\right)}{r_{\mathrm{eff}}\left(r_{0}\right)}, \quad r_{\mathrm{eff}}(r)=\frac{\left|r_{c}\right|}{1-\exp \left(-\left|r_{c}\right| / r\right)},
\end{gathered}
$$

because in highly polar solvents the survival probability of charged geminate pairs $\widetilde{\Omega}^{c}\left(t, r_{0}\right)$ is kinetically identical to the corresponding value $\widetilde{\Omega}\left(t, r_{0}\right)$ without the Coulomb interaction. 
3.2. Hopping Motion of Scavengers. When calculating the bulk reaction kinetics, in this subsection we shall take that $C$ reactants (just as $B$ ) move by jumps of infinite length:

$$
\widehat{\mathcal{L}}_{B C}=-\left(\tau_{B}^{-1}+\tau_{C}^{-1}\right) \hat{I} .
$$

The choice of this model of motion excludes recontacts determined by the kinematics of reactants approach thus reducing the value of time correlations in the system.

The model involving jumps of infinite length describes the so-called hopping mechanism of reactions. Physically it is realized when a reactant reaches the reaction zone in one jump. In this case the rate constant is equal to the product of the frequency of jumps and the reaction volume. Being an obvious alternative to diffusion mechanism, the hopping mechanism was first proposed in papers [36, 37] to describe luminescence impurity quenching experiments in solid solutions. For liquid phase reaction it is developed in papers [38-42] and was first found experimentally in excess electron capture reactions in nonpolar liquids [43].

The model of the "black" ball of the radius $R_{B C}$ insight of wich the reaction proceeds instantaneously is used for the calculation of the bulk kinetics $N(t)$. For the hopping mechanism this model is the analog of the commonly used "black" sphere model. In this case the initial nonstationary static stage of the kinetics turns into a point ("instantaneous quenching" [57]), and the bulk reaction proceeds exponentially. Calculations with $\hat{\mathcal{L}}_{B C}$ in form (29) yield

$$
N(t)=\exp \left[-\xi-\xi\left(\tau_{B}^{-1}+\tau_{C}^{-1}\right) t\right]
$$

where the dimensionless parameter $\xi=[C] V$ (the density parameter) appears. It is equal to the average number of acceptors in the reaction zone of the volume $V=(4 \pi / 3) R_{B C}^{3}$. Here we neglect the intrinsic volume of reactants and refer to long-range reactions.

Kinetics (30) consists of two cofactors. The first cofactor, $e^{-\xi}$, defines the depth of nonstationary stage and is the probability that at the initial instant of time there are no acceptors in the reaction volume. The second co-factor describes the exponential decay at a constant rate equal to the product of the total frequency of jumps $\tau_{B}^{-1}+\tau_{C}^{-1}$ and the average number of acceptors $\xi$ entering the reaction zone. Note that in the limit of immobile $B$ reactant $\left(\tau_{B} \rightarrow\right.$ $\infty) N(t)$ tends to the exact many-particle kinetics of the target problem $\tilde{N}(t)$ that for the hopping motion of scavengers is

$$
\tilde{N}(t)=\exp \left(-\xi-\xi \frac{t}{\tau_{C}}\right)
$$

Passing to experimentally measured quantities of the "scavenger problem", note that for both approximate theories the expression for $B$ reactant decay kinetics is obtained immediately after substitution of (24) and (30) in formulae (9) and (15), while calculation of the accumulation kinetics of geminate reaction products calls in addition for integration over time in (8) and (14). Nevertheless, we easily derive for the hopping motion of scavengers:

$$
\mathcal{P}_{P}^{s p}(t)=e^{-\xi} \mathcal{P}_{P}^{c t}(t), \quad \mathcal{P}_{B}^{s p}(t)=e^{-\xi} \mathcal{P}_{B}^{c t}(t)
$$

The above relations show that in the microscopic model chosen phenomenological theory (8), (9) becomes kinetically identical to the result of superposition approximation (14), (15).

The explicit expression for the accumulation kinetics of geminate reaction product in superposition approximation is deduced by substitution of (22), (30) in (14) and integration:

$$
\mathcal{P}_{P}^{s p}\left(t, r_{0}\right)=\frac{R_{A B}}{r_{0}} e^{-\xi} \varphi(\eta, \gamma+1, \tau),
$$

where $\gamma=\xi\left(\tau_{B} / \tau_{C}+1\right)$ and $\tau=t / \tau_{B}$ are bulk reaction rate and time in $\tau_{B}$ units, respectively.

Formula (33) involves the function $\varphi(\eta, x, \tau)$ :

$$
\begin{array}{r}
\varphi(\eta, x, \tau)=\frac{1}{2}\left\{e^{-\sqrt{\eta x}} \operatorname{erfc}\left(\sqrt{\frac{\eta}{4 \tau}}-\sqrt{x \tau}\right)\right. \\
\left.+e^{\sqrt{\eta x}} \operatorname{erfc}\left(\sqrt{\frac{\eta}{4 \tau}}+\sqrt{x \tau}\right)\right\},
\end{array}
$$

which, in accordance with the physical meaning of the product accumulation kinetics, is a monotonically increasing function that attains its steady-state value at rather long times:

$$
\varphi(\eta, x, \tau \longrightarrow \infty)=\exp (-\sqrt{\eta x})
$$

This gives the expression for the ultimate probability of geminate reaction in the presence of bulk acceptors:

$$
\mathcal{P}_{P}^{s p}\left(t \longrightarrow \infty, r_{0}\right)=\frac{R_{A B}}{r_{0}} e^{-\xi} \exp (-\sqrt{\eta(\gamma+1)}) .
$$

Comparison between (36) and (26) shows that the presence of bulk acceptors leads to decrease in the product yield of geminate reaction. The kinetics of $B$ reactant $\mathcal{P}_{B}^{s p}$ in accordance with the independence of geminate and bulk reactions in superposition approximation mentioned above is defined by (15), (24), and (30).

3.3. Diffusion Motion of Scavengers. The more realistic model, we shall take here for the bulk reaction that $C$ reactants move by continual diffusion with the diffusion coefficient $D_{C}$, while $B$ reactant (excess electron), as before, moves by infinite jumps with the mean time $\tau_{B}$ between them:

$$
\widehat{\mathcal{L}}_{B C}=D_{C} \nabla_{\vec{r}}^{2}-\tau_{B}^{-1} \hat{I} .
$$

During this time interval $\tau_{B}$ between jumps the bulk reaction develops due to acceptors diffusion to immobile electron (the "target problem" kinetics $\tilde{N}(t)$ ), while a regular very large electron jump changes the acceptors surroundings in an uncorrelated way, and the process is repeated until the twoscale bulk kinetics $N(t)$ is formed.

The model of a "black" ball of the radius $R_{B C}$ is used for further calculations. As in the previous subsection, in this case the initial nonstationary static stage of the kinetics turns into a point ("instantaneous quenching" [57]). It is obvious 
that for $\tau_{B}^{-1}=0$ the "target problem" kinetics $\tilde{N}(t)$ proceeds as the well-known Smoluchowski kinetics [53]:

$$
\tilde{N}(t)=\exp \left[-[C]\left(V+\tilde{k} t+2 \sqrt{\frac{3}{\pi} V \tilde{k} t}\right)\right], \quad \tilde{k}=4 \pi R_{B C} D_{C},
$$

where $V=(4 \pi / 3) R_{B C}^{3}$ is the reaction zone volume. The density parameter $\xi=[C] V$ equal to the average number of acceptors in the reaction zone of the volume $V$ can be introduced as in hopping kinetics (30).

The first co-factor of (38), $e^{-\xi}$, is the same as in (30), the second co-factor describes the exponential decay with the diffusion rate constant $\widetilde{k}$, and the last one is the diffusion nonstationary correction. In this subsection we use the binary approximation of the bulk kinetics [58], which allows us to expand the nonstationary part of the kinetics with the accuracy of a linear term over acceptor concentration $[C]$ :

$$
\tilde{N}(t) \approx e^{-[C] \tilde{k} t}\left[1-\xi-2 \sqrt{\frac{3}{\pi} \xi[C] \tilde{k} t}\right] .
$$

The validity condition of formula (39) defines the binary time interval $[C] \tilde{k} t \ll \xi^{-1}$ in which time-dependent observable values will be considered.

The kinetics of the bulk reaction $N(t)$ (16) can be obtained by solving (19) with operator (37) in the "black" sphere limit. In the binary approximation it takes the form

$$
N(t) \approx e^{-[C] k t}\left[1-\xi-2 f\left(\frac{t}{\tau_{B}}\right) \sqrt{\frac{3}{\pi} \xi[C] \tilde{k} t}\right],
$$

where, in contrast to the case of diffusion mobilities of both reactants, stationary rate constant of the bulk two-scale reaction is

$$
k=\tilde{k}+\frac{V}{\tau_{B}}+\sqrt{3 \tilde{k} \frac{V}{\tau_{B}}}
$$

and $f(\tau)$ is a function decreasing from unity to zero:

$$
f(\tau)=\frac{1}{2}\left[e^{-\tau}+\left(\sqrt{\frac{\pi}{4 \tau}}+\sqrt{\pi \tau}\right) \operatorname{erf}(\sqrt{\tau})-\sqrt{\pi \tau}\right] .
$$

At the time $t \gg \tau_{B}$ it goes to its asymptotic $f(\tau) \approx \sqrt{(\pi / \tau)} / 4$, and the nonstationary part of bulk kinetics becomes time independent.

Stationary rate constant (41) is equal to the sum of diffusion rate constant $\tilde{k}(38)$, hopping rate constant $V / \tau_{B}$ [36-42] and the interference term appearing due to the intricate character of reactants approach. The presence of this term is a distinguishing feature of stationary rate constant (41) of the two-scale bulk reaction. It has been already obtained in [54]. The second feature, as follows from our result (40), consists in the decrease of the nonstationary part of the kinetics as compared to (39) owing to the decreasing function $f\left(t / \tau_{B}\right)$.
The expression for the accumulation kinetics of geminate reaction product in the superposition approximation is deduced by substitution of (22), (40) in (14) and integration

$$
\mathcal{P}_{P}^{s p}\left(t, r_{0}\right)=(1-\xi) \mathcal{P}_{P}^{(m) s p}\left(t, r_{0}\right)-\mathcal{P}_{P}^{(n) s p}\left(t, r_{0}\right),
$$

where the Markovian part of the kinetics corresponding to pure exponential term of (40) is:

$$
\mathcal{P}_{P}^{(m) s p}\left(t, r_{0}\right)=\frac{R_{A B}}{r_{0}} \varphi(\eta, \gamma+1, \tau), \quad \gamma=[C] k \tau_{B} .
$$

As in the previous subsection, the introduced parameter $\gamma$ is the bulk reaction rate in $\tau_{B}$ units. Using (41), it can be rewritten in our case of diffusion motion of acceptor as $\gamma=$ $\beta+\xi+\sqrt{3 \xi \beta}$, where $\beta=[C] \tilde{k} \tau_{B}$.

The non-Markovian part of the kinetics is defined by the integral

$$
\begin{aligned}
\mathcal{P}_{P}^{(n) s p}\left(t, r_{0}\right)= & \frac{R_{A B}}{r_{0}} \frac{\sqrt{3}}{\pi} \sqrt{\xi \eta \beta} \int_{0}^{(\gamma+1) \tau} \frac{d x}{x} \\
& \times \exp \left(-x-\frac{\eta(\gamma+1)}{4 x}\right) f\left(\frac{x}{\gamma+1}\right) .
\end{aligned}
$$

This integral is not taken analytically even in the stationary limit $\tau \rightarrow \infty$.

In accordance with (15), the kinetics $\mathcal{P}_{B}^{s p}$ of $B$ reactant is defined by the product of (24) and (40).

\section{Exactly Solvable Model}

The choice of the hopping mobility of an excess electron in the above microscopic description of a reacting system makes it possible to use the exactly solvable model of the "scavenger problem" formulated by the authors in [34]. It should be kept in mind that, since the exactly solvable many-particle model takes no account of a hard core in the description of geminate reaction, the microscopic model can be formulated with the "black" sphere with penetration instead of the one without it. This will not affect the results of the preceding section, because we always consider the case where the initial distance in a geminate pair exceeds the "black" sphere radius $\left(r_{0}>\right.$ $R_{A B}$ ); later on repulsive forces cannot manifest themselves due to the instantaneous decay of the pair on the approach of reactants to the distance $R_{A B}$.

Assuming the hopping motion of $B$ reactant, the exactly solvable many-particle model of the "scavenger problem" gives the recipe for the calculation of the quantities measured:

$$
\begin{gathered}
\dot{\mathcal{P}}_{P}(t)=K_{g}(\tau) \tilde{N}(\tau), \quad \mathcal{P}_{P}(t)=\int_{0}^{t} d \tau K_{g}(\tau) \tilde{N}(\tau), \\
\mathcal{P}_{B}(t)=\tilde{\Omega}(t) \tilde{N}(t) e^{-t / \tau_{B}}+\frac{1}{\tau_{B}} \int_{0}^{t} d \tau \tilde{N}(t-\tau) e^{-(t-\tau) / \tau_{B}} \mathcal{P}_{B}(\tau),
\end{gathered}
$$

where $K_{g}(t)$ is the pure geminate reaction rate defined in (10), (22), $\widetilde{\Omega}(t)$ is defined in (27), $\tilde{N}(t)$ is the exact 
many-particle survival probability of immobile $B$ reactant surrounded by bulk acceptors (see (31), (38)). The binary kinetics $N(t)$ (16) goes to it with $\tau_{B} \rightarrow \infty$.

In this limit of immobile $B$ particle recipes (46), (47) are reduced to

$$
\mathcal{P}_{P}(t)=\int_{0}^{t} d \tau \widetilde{K}_{g}(\tau) \tilde{N}(\tau) ; \quad \mathcal{P}_{B}(t)=\tilde{N}(t) \widetilde{\Omega}(t) .
$$

Comparison of (48) with (14), (15) shows that at $\tau_{B}=$ $\infty$ the superposition approximation coincides with the exact solution of the problem. Thus for immobile $B$ time correlations between geminate and bulk reactions are absent in the given model at any acceptor concentrations.

We begin the examination of time correlation influence with the accumulation kinetics analysis of geminate recombination products. Comparison between (46) and (14) shows that in the general case of mobile $B$ the rate of geminate reaction products formation $\dot{\mathcal{P}}_{P}(t)=K_{g}(t) \tilde{N}(t)$ cannot be expressed solely in terms of the kinetics of geminate and bulk reactions. It is the product of geminate reaction rate $K_{g}(t)$ and the above probability $\tilde{N}(t)$ formed only during the residence time of $B$ particle in a geminate pair before it jumps into the bulk. Thus here the use of pure bulk reaction kinetics $N(t)$ is physically unjustified.

4.1. Hopping Motion of Scavengers. To analyze errors brought about by superposition approximation at arbitrary mobility of $B$ reactants, we make calculations in the framework of the microscopic model chosen. Comparison of the kinetics $\tilde{N}(t)$ (31) with formula (30) shows that the bulk reaction kinetics $N(t)$ differs from $\tilde{N}(t)$ by the exponential factor:

$$
N(t)=\exp \left(-\xi \frac{t}{\tau_{B}}\right) \tilde{N}(t)
$$

Accordingly, the accumulation rate $\dot{\mathcal{P}}_{P}^{s p}(t)$ of geminate reaction products predicted by superposition approximation differs from the exact result in the same way. So the superposition approximation can decrease essentially the accumulation rate of geminate reaction products.

To find the explicit form of the exact many-particle accumulation kinetics $\mathcal{P}_{P}(t)$ of geminate reaction products, use formulae (46), (22), and (31). We have.

$$
\mathcal{P}_{P}\left(t, r_{0}\right)=\frac{R_{A B}}{r_{0}} e^{-\xi} \varphi(\eta, \nu+1, \tau), \quad \nu=\frac{\xi \tau_{B}}{\tau_{C}} .
$$

The introduced dimensionless parameter $v$ has the meaning of the bulk reaction rate with immobile $B$ reactant in units of $\tau_{B}$. It follows from its definition that the parameter $\gamma$ from (33) tends to $v$ with $\tau_{B}$ tending to $\infty$. The function $\varphi(\eta, x, \tau)$ is defined by formula (34). As expression (33) for $\mathcal{P}_{P}^{s P}$ coincides with (50) up to the substitution of $v$ for $\gamma$, the ultimate probability of geminate reaction can be obtained from (36):

$$
\mathcal{P}_{P}\left(t \longrightarrow \infty, r_{0}\right)=\frac{R_{A B}}{r_{0}} e^{-\xi} \exp (-\sqrt{\eta(\nu+1)}) .
$$

For small values of the parameter $\xi$ the result for the ratio of ultimate probabilities (36) and (51) is easily obtained:

$$
\frac{\mathcal{P}_{P}^{s p}\left(t \longrightarrow \infty, r_{0}\right)}{\mathcal{P}_{P}\left(t \longrightarrow \infty, r_{0}\right)}=\exp \left(-\frac{\xi}{2} \sqrt{\frac{\eta}{v+1}}\right), \quad \xi \ll 1 .
$$

Leaving the detailed analysis of deviations of superposition approximation from the exact solution for Section 5, note that, according to (52), these deviations may be considerable even at small values of $\xi$, on condition that the value of the parameter $\eta$ is sufficiently large.

The behaviour of the accumulation kinetics $\mathcal{P}_{P}\left(t, r_{0}\right)$ and accumulation rate $\dot{\mathcal{P}}_{P}\left(t, r_{0}\right)$ of geminate reaction products as compared to the results of superposition approximation is given in Figures 1 and 2. The superposition approximation decreases noticeably the ultimate probability of geminate reaction in the presence of scavengers, and, according to (49), overestimates the decay of $\dot{\mathcal{P}}_{P}^{s p}\left(t, r_{0}\right)$ as compared to the exact solution denoted by lines with circles.

Before passing to the decay kinetics $\mathcal{P}_{B}(t)$ of $B$ reactant, note that no radical distinctions should be expected at least at low concentrations of acceptors. The reason is that in the case of rather fast motion of $B$, correlations are essential, and the contribution of geminate reaction into complete kinetics is insignificant. When $B$ reactant moves slowly, and the contribution of geminate reaction is considerable, correlations in the system are negligible, and the superposition approximation is found to be suitable.

To calculate the explicit form of the exact many-particle kinetics of $B$ reactant, we solve (47) by a conventional method of the Laplace transformation using expressions (27) and (31) for $\widetilde{\Omega}\left(t, r_{0}\right)$ and $\tilde{N}(t)$. This gives

$$
\mathcal{P}_{B}\left(t, r_{0}\right)=N_{B}(t)\left[1-\frac{R_{A B}}{r_{0}} \varphi\left(\eta, e^{-\xi}, \tau\right)\right] .
$$

Here $N_{B}(t)$ is the exact many-particle kinetics of the bulk reaction representable as

$$
N_{B}(t)=\exp \left(-\xi-\xi \frac{t}{\tau_{C}}-\left(1-e^{-\xi}\right) \frac{t}{\tau_{B}}\right) \equiv \tilde{N}(t) N_{0}(t)
$$

Formula (54) is physically clear. The bulk reaction kinetics is defined by two independent processes. The first process described by $\tilde{N}(t)$ (31) is the reaction between mobile acceptors and immobile $B$ particle, while the second one, $N_{0}(t)$, defines the reaction of mobile $B$ particle with the ensemble of immobile acceptors. A regular very large jump of $B$ changes the environment of acceptors in an uncorrelated way, thus the rate of the second process is equal to the product of the frequency of jumps $1 / \tau_{B}$ and the manyparticle probability of acceptors entering the reaction zone, $1-e^{-\xi}$. At small parameters $\xi$ expansion (54) gives exactly the formula of binary kinetics (30) depending on a relative mobility of $B$ and $C$ reactants.

It is easily seen that the structure of expression (53) coincides with the structure defined by superposition approximation recipe (15). As in the case of immobile $B$ particle, the complete kinetics can be divided into a product of two 
cofactors. The first co-factor describes the many-particle kinetics of the bulk reaction $N_{B}(t)$ which is the solution of (47) in the absence of geminate recombination $(\widetilde{\Omega}(t) \equiv 1)$. Following the superposition approximation logic, the second co-factor may be considered to be the survival probability of some effective geminate pair making allowance for correlations with the bulk reaction:

$$
\Omega^{\mathrm{eff}}\left(t, r_{0}\right)=1-\frac{R_{A B}}{r_{0}} \varphi\left(\eta, e^{-\xi}, \tau\right) .
$$

This is also confirmed by the absence of the dependence $\Omega^{\mathrm{eff}}\left(t, r_{0}\right)$ on $C$ reactant mobility.

As one would expect, the influence of correlations disappears under infinite dilution of bulk scavengers: with $\xi \rightarrow 0$ expression (55) goes into (24). However, it is seen that the presence of acceptors in (55) results in the acceleration of the kinetics $\Omega^{\mathrm{eff}}\left(t, r_{0}\right)$ as compared to pure geminate kinetics $\Omega\left(t, r_{0}\right)$. Correspondingly, the stationary portion of nonreacting geminate pairs (the escape probability) is less than in (26):

$$
\Omega_{\infty}^{\mathrm{eff}}\left(r_{0}\right)=1-\frac{R_{A B}}{r_{0}} \exp \left(-\sqrt{\eta e^{-\xi}}\right)
$$

This means that in a hypothetical effective pair geminate recombination proceeds at a higher rate. Unlike (22), in such a pair the reaction rate may be represented as

$$
K_{g}^{\mathrm{eff}}\left(t, r_{0}\right)=\tilde{K}_{g}\left(t, r_{0}\right) \exp \left(-\frac{t}{\tau_{B}} e^{-\xi}\right) .
$$

This is paradoxical: taking account of time correlations with the competing bulk reaction accelerates the reaction in effective geminate pair, though it seems obvious that it must be retarded due to additional decay channel in effective acceptor surroundings. However, the paradox arises from the superposition approximation logic. The attempt to treat the exact kinetics $\mathcal{P}_{B}\left(t, r_{0}\right)$ as the product of the bulk kinetics and some independent effective geminate kinetics is physically unjustified.

Another interpretation of the rate $K_{g}^{\text {eff }}\left(t, r_{0}\right)(57)$ is given by the representation:

$$
K_{g}^{\mathrm{eff}}\left(t, r_{0}\right)=\frac{K_{g}\left(t, r_{0}\right) \tilde{N}(t)}{N_{B}(t)} .
$$

It shows that the increase in $K_{g}^{\text {eff }}\left(t, r_{0}\right)$ is related to defect of the superposition approximation that underestimates significantly geminate reaction product accumulation rate due to exponential difference between $N(t)$ and $\tilde{N}(t)$ (see (49)). The dependence of $K_{g}^{\text {eff }}\left(t, r_{0}\right)$ on the bulk reaction kinetics $N_{B}(t)$ suggests that geminate and bulk reactions are correlated in time. That is why the attempt to represent them as two independent processes leads to physical contradiction. Note that adequate description follows from the derivation of kinetic equations of the "scavenger problem" based on the application of general kinetic theory concepts to the exactly solvable many-particle problem [35].

The behaviour of the kinetics $\mathcal{P}_{B}\left(t, r_{0}\right)$ and $\mathcal{P}_{B}^{s p}\left(t, r_{0}\right)$ is given in Figure 3 for two values of the density parameter $\xi$.

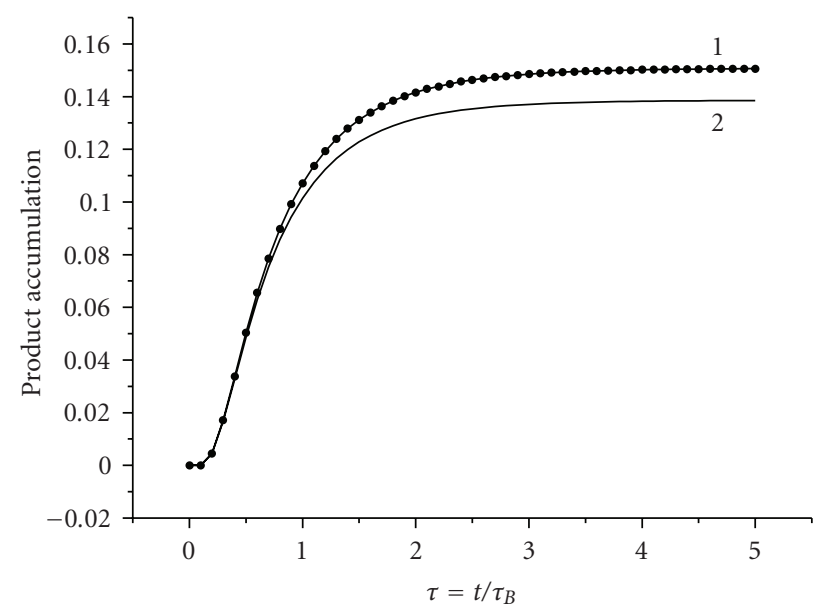

Figure 1: Exact accumulation kinetics $\mathcal{P}_{P}(t)-(1)$, and superposition approximation $\mathcal{P}_{P}^{s p}(t)-(2)$ for geminate reaction products in the presence of scavengers. $\xi=0.1, \eta=3, R_{A B} / r_{0}=0.95, \tau_{B} / \tau_{C}=$ 0.1 .

Note that relative deviations at small values of $\xi$ (curves 1,2 ) are not so great as for geminate reaction product accumulation. They are proportional to the density parameter. Increased distinctions beyond the limits of the binary interval (curves 3,4 ) are due to the many-particle nature of the kinetics $N_{B}(t)(54)$.

4.2. Diffusion Motion of Scavengers. For diffusion motion of scavengers comparison between binary formulae (39) and (40) shows that the bulk reaction kinetics $N(t)$ differs from $\tilde{N}(t)$ by the exponential multiplier due to the difference between $k$ and $\tilde{k}$, while the second power factor appears due to nonstationary parts of kinetics (39) and (40):

$$
\begin{array}{r}
\frac{N(t)}{\tilde{N}(t)}=\exp [-(\xi+\sqrt{3 \xi \beta}) \tau]\left[\frac{1-\xi-f(\tau) \tilde{n}(\tau)}{1-\xi-\tilde{n}(\tau)}\right], \\
\beta=[C] \tilde{k} \tau_{B} .
\end{array}
$$

Here $\beta$ and $\tau=t / \tau_{B}$ are diffusion reaction rate with immobile $B$ reactant and time in $\tau_{B}$ units, respectively. We introduce the notation for diffusion nonstationary part of kinetics (38), (39):

$$
\tilde{n}(\tau)=2 \sqrt{\frac{3 \xi \beta \tau}{\pi}} .
$$

Accordingly, the accumulation rate $\dot{\mathcal{P}}_{P}^{s p}(t)$ of geminate reaction products predicted by superposition approximation (14) differs from the exact result in the same way. As is seen, for diffusion motions of scavengers these distinctions become more significant because of the presence of extra root term in the exponent of (59).

To find the accumulation kinetics $\mathcal{P}_{P}(t)$ of geminate reaction products, use formulae (46), (22), and (39). We have:

$$
\mathcal{P}_{P}\left(t, r_{0}\right)=(1-\xi) \mathcal{P}_{P}^{(m)}\left(t, r_{0}\right)-\mathcal{P}_{P}^{(n)}\left(t, r_{0}\right),
$$




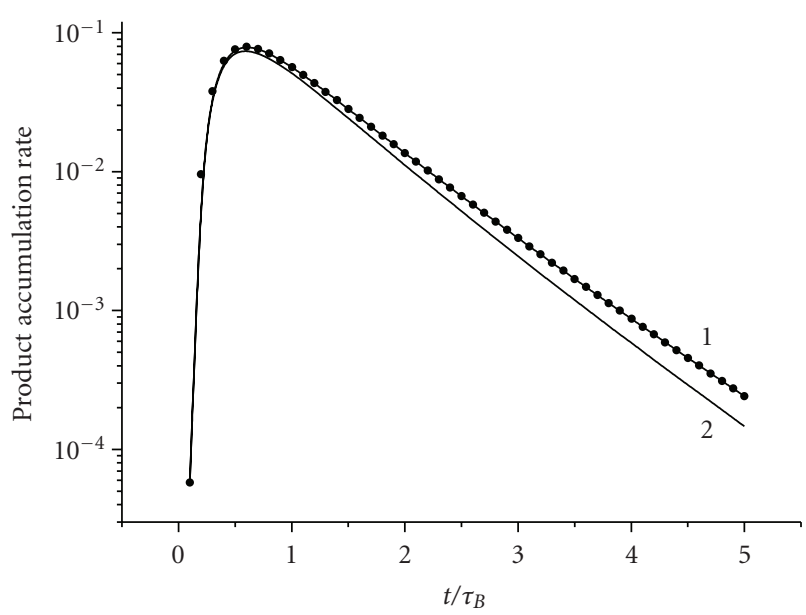

Figure 2: Exact product accumulation rate $\dot{\mathcal{P}}_{P}(t)-(1)$, and superposition approximation $\dot{\mathcal{P}}_{P}^{s p}(t)-(2)$. Parameters are the same as in Figure 1.

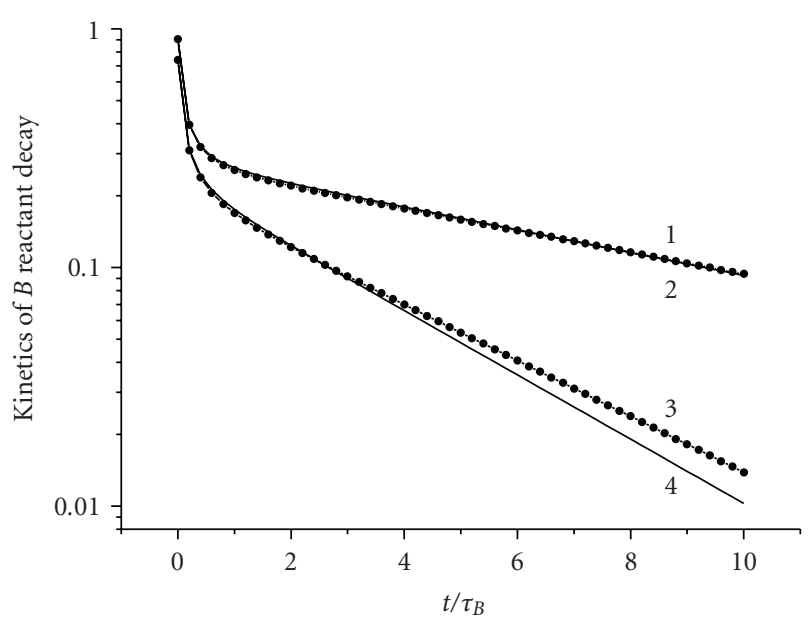

Figure 3: Exact kinetics $\mathcal{P}_{B}(t)-(1,3)$ and superposition approximation $\mathcal{P}_{B}^{s p}(t)-(2,4)$ for different values of $\xi: \xi=0.1-(1,2)$; $\xi=0.3-(3,4)$. Other parameters: $\eta=0.1, R_{A B} / r_{0}=0.95$, $\tau_{C} / \tau_{B}=0.1$.

where $\mathcal{P}_{P}^{(m)}$ is the contribution of the Markovian (exponential) term of (39) calculated in the previous section:

$$
\mathcal{P}_{P}^{(m)}\left(t, r_{0}\right)=\frac{R_{A B}}{r_{0}} \varphi(\eta, \beta+1, \tau)
$$

where the function $\varphi(\eta, x, \tau)$ is defined in (34). The value $\mathcal{P}_{P}^{(n)}\left(t, r_{0}\right)$ is determined by diffusion non-Markovian part of kinetics (39) and is equal to

$\mathcal{P}_{P}^{(n)}\left(t, r_{0}\right)=\frac{R_{A B}}{r_{0}} \frac{\sqrt{3}}{\pi} \sqrt{\xi \eta \beta} \int_{0}^{(\beta+1) \tau} \frac{\mathrm{d} x}{x} \exp \left(-x-\frac{\eta(\beta+1)}{4 x}\right)$.
In the limit $t \rightarrow \infty$ the integral in (63) goes to the Bessel function. In this limit, using (35), we can rewrite (61) in an analytical form:

$$
\begin{aligned}
\mathcal{P}_{P}\left(\infty, r_{0}\right)=\frac{R_{A B}}{r_{0}}[ & (1-\xi) e^{-\sqrt{\eta(\beta+1)}} \\
& \left.-\frac{2 \sqrt{3}}{\pi} \sqrt{\xi \eta \beta} K_{0}(\sqrt{\eta(\beta+1)})\right],
\end{aligned}
$$

where $K_{0}(x)$ is the modified Bessel function of a zero order [59].

The behaviour of the exact accumulation kinetics $\mathcal{P}_{P}(t)$ (61)-(63) and superposition approximation $\mathcal{P}_{P}^{s p}(t)(43)-$ (45) is shown in Figure 4.

The ratio of (43) and (61) at large $\tau$ and small $\xi$ is recast as

$$
\frac{\mathcal{P}_{P}^{s p}\left(\infty, r_{0}\right)}{\mathcal{P}_{P}\left(\infty, r_{0}\right)}=\exp \left[-\frac{\xi}{2} \sqrt{\frac{\eta}{\beta+1}}\left(1+\sqrt{\frac{3 \beta}{\xi}}\right)\right] \Theta, \quad \xi \ll 1 .
$$

The first exponential factor in (65) is defined by the ratio between Markovian contributions (44) and (62), while $\Theta$ specifies the relation of non-Markovian parts of geminate product:

$$
\Theta=\frac{1-\xi-\mathcal{P}_{P}^{(n) s p}\left(\infty, r_{0}\right) / \mathcal{P}_{P}^{(m) s p}\left(\infty, r_{0}\right)}{1-\xi-\mathcal{P}_{P}^{(n)}\left(\infty, r_{0}\right) / \mathcal{P}_{P}^{(m)}\left(\infty, r_{0}\right)} .
$$

From (65) as well as from (52) of the previous subsection it follows that even at small $\xi$ the result of superposition approximation can decrease essentially the ultimate probability of geminate recombination in the presence of scavengers in comparison with the exact solution if $\eta$ is rather large.

To calculate the decay kinetics $\mathcal{P}_{B}(t)$ of $B$ reactant, we solve (47) by a conventional method of the Laplace transformation using expressions (27) and (39) for $\widetilde{\Omega}\left(t, r_{0}\right)$ and $\tilde{N}(t)$. This equation has a many-particle character. The correct binary approximation in the limit of ultrafast geminate reaction has to turn into the product of the escape probability and bulk kinetics: $\mathcal{P}_{B}\left(t, r_{0}\right)=\Omega_{\infty}\left(r_{0}\right) N(t)$ [35]. Taking into account this requirement, we obtain that

$$
\mathcal{P}_{B}\left(t, r_{0}\right)=(1-\xi) \mathcal{P}_{B}^{(m)}\left(t, r_{0}\right)-\mathcal{P}_{B}^{(n)}\left(t, r_{0}\right),
$$

where the Markovian part of the kinetics can be represented as

$$
\begin{array}{r}
\mathcal{P}_{B}^{(m)}\left(t, r_{0}\right)=e^{-[C] k t}\left[1-\frac{R_{A B}}{r_{0}} \varphi(\eta, \alpha, \tau)\right], \\
\alpha=1-\xi-\sqrt{3 \xi \beta} .
\end{array}
$$

This result coincides with the corresponding result of (53) at a small value of $\xi$ except the parameter $\alpha$. At large $t$ expression (68) goes to the following:

$$
\begin{aligned}
\mathcal{P}_{B}^{(m)}\left(t, r_{0}\right) & \simeq e^{-[C] k t}\left[1-\frac{R_{A B}}{r_{0}} e^{-\sqrt{\alpha \eta}}\right] \\
& =e^{-[C] k t} \Omega_{\infty}^{\mathrm{eff}}\left(r_{0}\right), \quad t \gg \tau_{B} .
\end{aligned}
$$




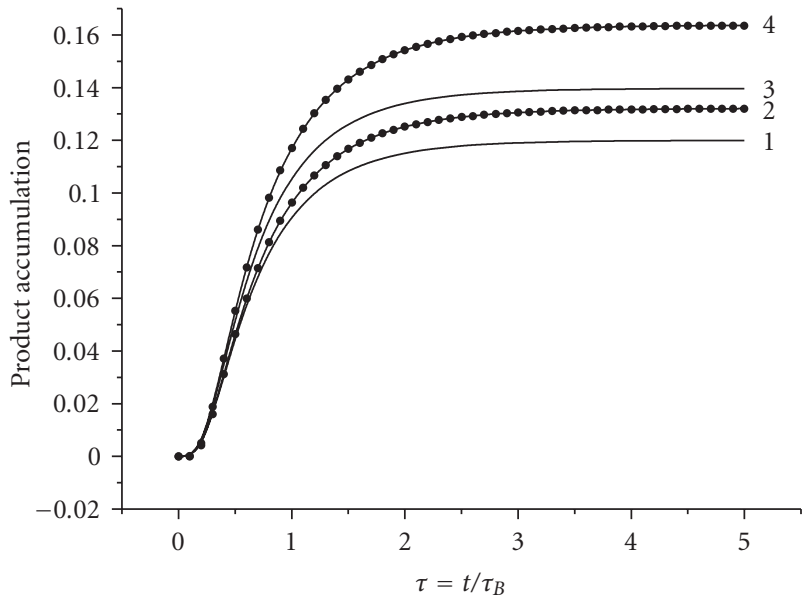

Figure 4: Exact accumulation kinetics $\mathcal{P}_{P}(t)-(2)$, its Markovian part $\mathcal{P}_{P}^{(m)}(t)-(4)$, and superposition approximation $\mathcal{P}_{P}^{s p}(t)-(1)$, its Markovian part $\mathcal{P}_{P}^{(m) s p}(t)-(3)$ for geminate reaction products in the presence of diffusing scavengers. $\xi=0.1, \eta=3, R_{A B} / r_{0}=0.95$, $\tau_{B} / \tau_{C}=3 D_{C} \tau_{B} / R_{B C}^{2}=0.3$.

The non-Markovian part of the kinetics can be represented through quadrature of elementary functions.

It is interesting to compare $\mathcal{P}_{B}$ and $\mathcal{P}_{B}^{s p}(15),(24),(40)$ at a long time when $B$ reactant leaves a geminate pair for the bulk. In this case the expressions for these kinetics are simplified, and we obtain the relation:

$$
\begin{array}{r}
\frac{\mathcal{P}_{B}^{s p}\left(t, r_{0}\right)}{\mathcal{P}_{B}\left(t, r_{0}\right)} \simeq \frac{\Omega_{\infty}}{\Omega_{\infty}^{\mathrm{eff}}}\left(1-\frac{R_{A B}}{r_{0}} \frac{\left(e^{-\sqrt{\alpha \eta}}-J(\alpha \eta)\right) \sqrt{3 \xi \beta / \alpha}}{(1-\xi-\sqrt{3 \xi \beta / 2}) \Omega_{\infty}^{\mathrm{eff}}}\right)^{-1}, \\
t \gg \tau_{B},
\end{array}
$$

where $\Omega_{\infty}$ is the escape probability defined by (26), and $J(x)$ is the following quadrature:

$$
J(x)=\frac{2}{\sqrt{\pi}} \int_{0}^{\infty} \mathrm{d} y \sqrt{y} \exp (-y) \operatorname{erfc}\left(\sqrt{\frac{x}{4 y}}\right) .
$$

It easy to see that in (70) the factor $\Omega_{\infty} / \Omega_{\infty}^{\text {eff }}$ is defined by the ratio of the Markovian parts of $\mathcal{P}_{B}^{s p}$ and $\mathcal{P}_{B}$, while the multiplier between the brackets is the relation of the nonMarkovian parts of the kinetics.

\section{Analysis of Deviations}

To describe the distinctions between the exact solution and superposition approximation, we introduce relative deviations of the quantities measured:

$$
\begin{gathered}
\Delta_{P \text { dot }}=\frac{\left(\dot{\mathcal{P}}_{P}-\dot{\mathcal{P}}_{P}^{s p}\right)}{\dot{\mathcal{P}}_{P}}, \quad \Delta_{P}=\frac{\left(\mathcal{P}_{P}-\mathcal{P}_{P}^{s p}\right)}{\mathcal{P}_{P}}, \\
\Delta_{B}=\frac{\left(\mathcal{P}_{B}-\mathcal{P}_{B}^{s p}\right)}{\mathcal{P}_{B}} .
\end{gathered}
$$

Taking into consideration that comparison is worthwhile in the region of small concentrations, in calculating relative deviations the exact many-particle quantities should be expanded in terms of a small parameter $\xi$. This imposes restrictions on the time interval of the comparison. In particular, the binary interval where the approximation linear in $\xi$ is defined by the quadratic term smallness condition: $[C] k t \ll \xi^{-1}$. This criterion can be defined more precisely for our exactly solvable model. Note that, in fact, quadratic corrections are determined both by the expansion of the many-particle rate constant nonlinearly dependent on acceptor concentration and by the expansion of the nonstationary part of the kinetics. The smallness condition for corrections of the first type is determined by the inequality $[C](k-\tilde{k}) t \ll \xi^{-1}$, while the smallness of the second type corrections requires (as is seen from (39)) that the condition $[C] \tilde{k} t \ll \xi^{-1}$ be fulfilled. Thus the binary time interval where comparison of the theories will be made is given by the criterion:

$$
t \ll t_{\mathrm{bin}}=\xi^{-1} \min \left\{([C](k-\tilde{k}))^{-1},([C] \tilde{k})^{-1}\right\} .
$$

The most considerable deviation exponentially increasing with time takes place for the first quantity in (72) whatever the relation between geminate and bulk reactions. Substituting the values of the rates $\dot{\mathcal{P}}_{P}$ and $\dot{\mathcal{P}}_{P}^{s p}$ for the hopping and diffusion models of scavenger motion in $\Delta_{P \text { dot }}$, we have, correspondingly,

$\Delta_{P \operatorname{dot}}$

$$
= \begin{cases}1-e^{-\xi \tau}, & \tau \ll \xi^{-2}, \\ 1-e^{-(\xi+\sqrt{3 \xi \beta}) \tau}\left[\frac{1-\xi-f(\tau) \tilde{n}(\tau)}{1-\xi-\tilde{n}(\tau)}\right], & \tau \ll \frac{1}{\xi(\xi+\sqrt{3 \xi \beta})} .\end{cases}
$$

It is seen that exponential deviation $\dot{\mathcal{P}}_{P}^{s p}$ from $\dot{\mathcal{P}}_{P}$ leads to the fact that even at half-decay times $t \approx \tau_{C} / \xi$ of the kinetics $\tilde{N}(t)$ (31) the relative deviation $\Delta_{P \text { dot }}$ may differ from unity just by several tens percent. In particular, for the hopping model of scavenger motion $\Delta_{P \text { dot }}=0.63 ; 0.86 ; 0.95$ for $\tau_{B}=\tau_{C}$, $\tau_{B}=\tau_{C} / 2, \tau_{B}=\tau_{C} / 3$, respectively. For diffusion model of scavenger motion these distinctions are even more essential. It should be noted that $\Delta_{P \text { dot }}$ does not depend on initial distance in geminate pair $r_{0}$. Thus, (74) are correct for any distribution of initial distance $P\left(r_{0}\right)$.

The behaviour of the relative deviation $\Delta_{P \text { dot }}$ is given in Figure 5. It is evident that deviations are already very large even at the very beginning of a binary time interval both for diffusion (line 2) and for hopping (line 3) motion of scavengers. The second power factor of (49) slightly decreases the deviation in comparison with the Markovian exponential part of the kinetics designated in Figure 5 by line 1. Nevertheless the deviation for diffusion remains larger than that for the hopping motion of acceptors (the first line in $(74))$. 


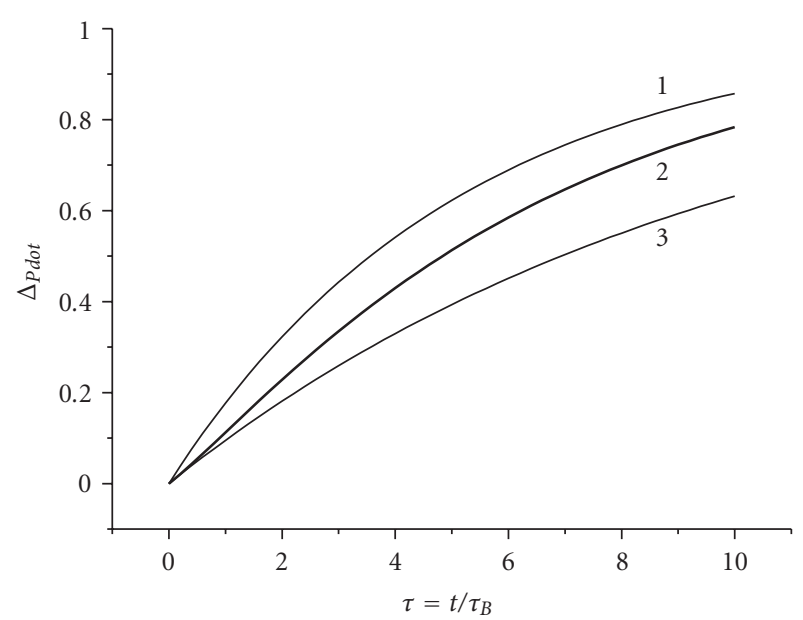

FIGURE 5: Relative deviation $\Delta_{P \text { dot }}$ for diffusion (2) and for hopping motion of scavengers (3). Line (1) is the Markovian part $\Delta_{P \operatorname{dot}}^{(m)}$ of deviation for the diffusion case. Parameters: $\xi=0.1, \tau_{B} / \tau_{C}=$ $3 \tau_{B} D_{C} / R_{B C}^{2}=0.3$.

Consider the behaviour of the function $\Delta_{P}$ that describes correlations arising in the course of geminate recombination product accumulation. Since time correlations accumulate with time, and superposition approximation takes no account of this process, the quantity $\Delta_{P}$ increases monotonically with time and attains its steady-state value $\Delta_{P}^{s}$ rather rapidly. The behaviour of the relative deviation $\Delta_{P}$ of accumulation kinetics (50) and (61) as compared to the results of superposition approximation (33) and (43) is given in Figure 6. As we can see, for diffusion model relative deviations $\Delta_{P}$ (line 2) and its Markovian version $\Delta_{P}^{(m)}=1-\mathcal{P}_{P}^{(m) s p} / \mathcal{P}_{P}^{(m)}$ (line 1) grow up with $\tau$ and rapidly go to stationary values. The non-Markovian part of the kinetics decreases the relative deviation but nevertheless, as in Figure 5, it remain larger than for the model of the hopping motion of scavengers (line 3 ).

Using the expansion of the functions $\mathcal{P}_{P}^{s p}\left(\infty, r_{0}\right) /$ $\mathcal{P}_{P}\left(\infty, r_{0}\right)$ from (52) and (65) for hopping and diffusion models of scavenger motion at small values of the parameter $\xi$, we have the relation for stationary relative deviations:

$$
\Delta_{P}^{s}= \begin{cases}1-\exp \left(-\frac{\xi}{2} \sqrt{\frac{\eta}{v+1}}\right), & \text { hopping, } \\ 1-\exp \left(-\frac{\xi}{2} \sqrt{\frac{\eta}{\beta+1}}\left(1+\sqrt{\frac{3 \beta}{\xi}}\right)\right) \Theta, & \text { diffusion, }\end{cases}
$$

where the function $\Theta$ is given by relation (66).

It is easy to see that at the start at the contact $\left(r_{0}=\right.$ $R_{A B}, \eta=0$ ) the function $\Delta_{P}^{s}$ is equal to zero. Then, as $\eta$ increases, $\Delta_{P}^{s}$ rises monotonically gradually approaching its asymptotic value equal to unity. However, it should be taken into account that the use of (75) is justified only in the case where the attainment of steady-state values occurs in the binary interval. This imposes definite restrictions on the values of the parameter $\eta$ essential in the analysis of

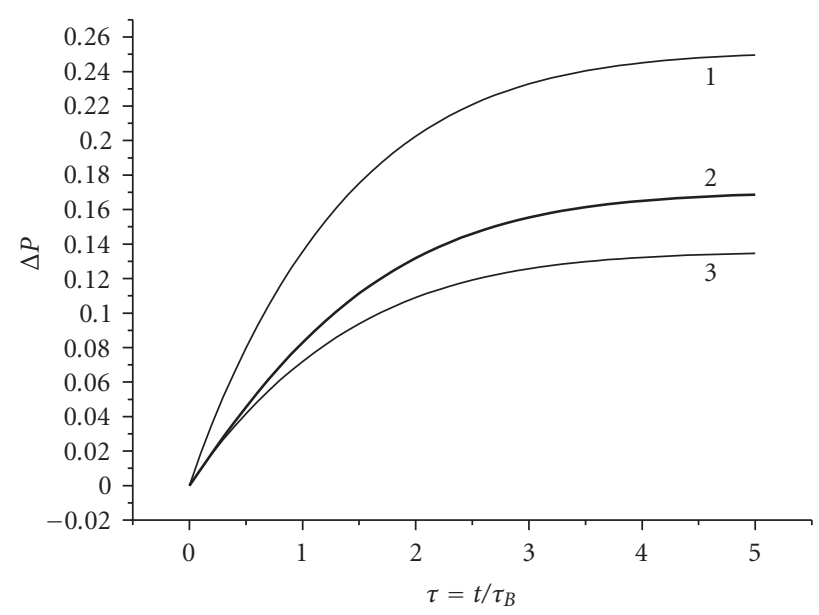

FIgURE 6: Relative deviation $\Delta_{P}(2)$ and its Markovian part $\Delta_{P}^{(m)}$ (1) for diffusing scavengers in comparison with $\Delta_{P}$ for hopping scavengers (3). Parameters: $\xi=0.1, \eta=10, R_{A B} / r_{0}=0.95$, $\tau_{B} / \tau_{C}=3 \tau_{B} D_{C} / R_{B C}^{2}=0.3$.

$\Delta_{P}^{s}$. Indeed, the use of the binary kinetics in (14) calls for the smallness of the characteristic decay time $\tau_{g}(23)$ of a geminate pair as compared to the binary interval $\left(\tau_{g} \ll t_{\text {bin }}\right)$. As a result, at $\eta \gg 1$, when $\tau_{g}=3 \sqrt{\tilde{\tau}_{g} \tau_{B}}$, an insignificant upper bound is set on the parameter $\eta$. In the hopping case this restriction is defined by the inequality $\sqrt{\eta} \ll \xi^{-2}$. However, this restriction is no barrier to the realization of considerable relative deviations $\Delta_{P}^{s}$ possible on condition that the exponent index in (75) is greater than unity. For the hopping model of scavengers motion this condition is defined by the inequality:

$$
\frac{\xi}{2} \sqrt{\frac{\eta}{\nu+1}}=\frac{\xi}{6} \frac{\tau_{g}}{\tau_{B}} \frac{1}{\sqrt{1+\xi \tau_{B} / \tau_{C}}}>1
$$

For acceptors at rest $\left(\tau_{C} \rightarrow \infty\right)$ condition (76) is realized at

$$
\xi \frac{\tau_{g}}{\tau_{B}}>1
$$

Physically this means that geminate pair lifetime is long enough for the bulk reaction (proceeding at the rate $\xi / \tau_{B}$ ) to reach considerable depth. Time correlations in this case are maximum. When $C$ reactant starts moving, correlations decrease. In the region $\xi \tau_{B} \ll \tau_{C} \ll \tau_{B}$, where the bulk reaction rate is $\xi / \tau_{C}$ (since this rate can be calculated neglecting $B$ reactant motion), but the bulk reaction depth for the time $\tau_{B}(\nu \ll 1)$ is small, correlations become essential under more rigid restrictions:

$$
\xi \frac{\tau_{g}}{\tau_{C}}>\frac{\tau_{B}}{\tau_{C}} \gg 1
$$

As the motion of $C$ reactants becomes more active $\left(\tau_{C} \ll\right.$ $\left.\xi \tau_{B}\right)$, the region of essential deviations is reached under even more strict conditions:

$$
\xi \frac{\tau_{g}}{\tau_{C}}>\frac{\tau_{B}}{\tau_{C}} \sqrt{\xi \frac{\tau_{B}}{\tau_{C}}} \gg \frac{\tau_{B}}{\tau_{C}} \gg 1 .
$$


For diffusion motion of scavengers the condition of large relative deviations is as follows:

$$
\frac{\xi}{2} \sqrt{\frac{\eta}{\beta+1}}\left(1+\sqrt{\frac{3 \beta}{\xi}}\right)=\frac{\xi}{6} \frac{\tau_{g}}{\tau_{B}} \frac{\left(1+\sqrt{3 \tau_{B} / \tau_{C}}\right)}{\sqrt{1+\xi \tau_{B} / \tau_{C}}}>1,
$$

where the mean residence time $\tau_{C}=R_{B C}^{2} /\left(3 D_{C}\right)$ of diffusing scavengers in the reaction zone is introduced. Obviously, with $\tau_{C} \rightarrow \infty$ inequality (80), just as (76), holds subject to condition (77). However, the situation reverses when diffusion of scavengers becomes so rapid that the mean residence time $\tau_{C}$ lies in the interval $\xi_{B} \ll \tau_{C} \ll \tau_{B}$. Then the second term between the brackets in (80) becomes decisive, and this equality may be written as

$$
\xi \frac{\tau_{g}}{\tau_{C}}>\sqrt{\frac{\tau_{B}}{\tau_{C}}} \gg 1
$$

It is seen that restriction (81) is much less rigid than (77). However, in this range of parameters the binary interval boundary for diffusion motion of scavengers is already determined by the smallness condition of non-Markovian corrections (the second term in (73)). Thus a more rigid upper bound on the value of the parameter $\eta$ is set: $\sqrt{\eta} \ll$ $(\xi \beta)^{-1}$. This is equivalent to the condition $\xi \tau_{g} / \tau_{C} \ll \xi^{-1}$. This inequality does not prevent the fulfillment of the realization condition of large relative deviations (80), though it sets the upper bound on this value. Thus condition (81) takes the form:

$$
\xi^{-1} \gg \xi \frac{\tau_{g}}{\tau_{C}}>\sqrt{\frac{\tau_{B}}{\tau_{C}}} \gg 1
$$

Finally, when the motion of scavengers becomes dominant $\left(\tau_{C} \ll \xi \tau_{B}\right)$, condition $(80)$ is met in the following restricted interval:

$$
\xi^{-1} \gg \xi \frac{\tau_{g}}{\tau_{C}}>\sqrt{\xi} \frac{\tau_{B}}{\tau_{C}} \gg 1
$$

Obviously, large relative deviations can be realized only on condition that the right and the left boundaries do not overlap: $\tau_{C} \gg \xi^{3 / 2} \tau_{B}$. With progressively decreasing $\tau_{C}$ the upper and the lower boundaries in inequality (83) approach one another thus eliminating the possibility of the realization of considerable relative deviations in the limit of dominating motion of scavengers. In the limit of infinitely high mobility of acceptors $\left(\tau_{C} \rightarrow 0\right)$ the difference between $N(t)$ and $\tilde{N}(t)$ vanishes, so the superposition approximation coincides with the exact solution, and the relative deviation tends to zero.

Thus conditions (77)-(79) and (81)-(83) show that correlations decrease with increasing intensity of $C$ reactants motion. It should be noted that in the region where deviations are small (reversal of inequalities (76) and (80)) they are still linear in $\xi$, that is, measurable in the framework of the binary approach. The relative deviation $\Delta_{P}^{s}$ as a function of parameter $\eta$ in the case of dominate electron motion $\left(\tau_{C} \gg \tau_{B}\right)$ is shown in Figure 7 . It is readily seen that taking into account the non-Markovian part of deviation defined by the factor $\Theta$ in (52) decreases noticeably the values

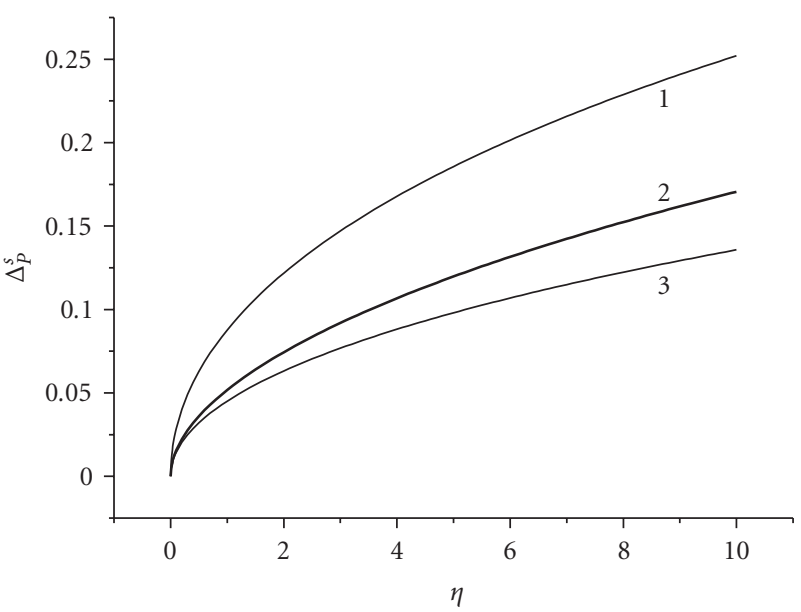

Figure 7: Relative deviation $\Delta_{P}^{s}$ defined by (75) for hopping (3) and for diffusing (2) scavengers versus $\eta=\tilde{\tau}_{g} / \tau_{B}$. Line (1) is the Markovian part of (2). Parameters: $\xi=0.1, \tau_{B} / \tau_{C}=3 \tau_{B} D_{C} / R_{B C}^{2}=$ 0.3 .

of $\Delta_{P}^{s}$ for diffusion model (line 2) in comparison with pure Markovian part (line 1) including only the first exponential part of relation (75). The corresponding deviation for the hopping acceptors motion (line 3 ) lies everywhere below.

Now we pass to the analysis of the function $\Delta_{B}$. Since the superposition approximation retards the decay kinetics of $B$ reactant as compared to the exact solution, this function is everywhere negative, except the initial instant of time. For the model of the hopping motion of scavengers in the binary interval it is of a simplest form:

$$
\Delta_{B}=1-\frac{\left[1-\left(R_{A B} / r_{0}\right) \varphi(\eta, 1, \tau)\right]}{\left[1-\left(R_{A B} / r_{0}\right) \varphi(\eta, 1-\xi, \tau)\right]} .
$$

We will not give similar expression for the model of diffusion motion of scavengers due to its cumbersome form. As shown below it behaves similarly to (84). One can readily see that function (84), though always negative, attains rapidly its steady-state value $\Delta_{B}^{s}$ defined by asymptotic (35) of the function $\varphi(\eta, x, \tau)$. In the range of small values of $\xi$ the expression for $\Delta_{B}^{s}$ can be brought to the form

$$
\Delta_{B}^{s}=-\frac{R_{A B}}{r_{0}} \frac{e^{-\sqrt{\eta}}\left(e^{\xi \sqrt{\eta} / 2}-1\right)}{1-\left(R_{A B} / r_{0}\right) e^{-\sqrt{\eta}(1-\xi / 2)}} .
$$

Examine the behaviour of the function $\Delta_{B}^{s}$ (85). If a geminate pair is formed not at the contact $\left(r_{0}>R_{A B}\right)$, then under variation of the parameter $\eta$ from 0 to $\infty$ this function begins with zero, passes through the minimum in the area $\eta<1$, and then smoothly tends to zero again with increasing $\eta$. The closer the starting point $r_{0}$ to the reaction zone, the sharper and deeper the minimum, and the greater its shift to the area of small values of $\eta$. In the region of the extremum $\eta<1$ expression (85) for $\Delta_{B}^{s}$ may be simplified:

$$
\Delta_{B}^{s}=-\frac{\xi}{2} \frac{R_{A B}}{r_{0}} \frac{\sqrt{\eta}}{e^{\sqrt{\eta}}-\left(R_{A B} / r_{0}\right)(1+\xi \sqrt{\eta} / 2)} .
$$


Function (86) has the minimum at the point $\eta_{m}$ determined by a transcendental equation:

$$
\frac{R_{A B}}{r_{0}} e^{-\sqrt{\eta_{m}}}=1-\sqrt{\eta_{m}}
$$

This equation has the only root lying in the interval $0 \leq \eta_{m}<$ 1. Substituting (87) in (86), we have with the accuracy up to the main order in $\xi$ :

$$
\min \Delta_{B}^{s} \simeq-\frac{\xi}{2}\left(1-\sqrt{\eta_{m}}\right) .
$$

The most essential deviations $\left|\Delta_{B}^{s}\right| \simeq \xi / 2$ arise at $\eta_{m} \ll 1$. Examining the function $\Delta_{B}^{s}$ in this interval, we easily obtain the analytical expression for the minimum:

$$
\eta_{m} \approx 2\left(1-\frac{R_{A B}}{r_{0}}\right) \ll 1 .
$$

As follows from (88), (89), the greatest deviation is realized in the case where a geminate pair is formed immediately near the contact. The definition of the parameter $\eta$ makes it possible to estimate the thickness of the layer the initial start from which leads to maximum time correlations:

$$
r_{0}-R_{A B} \simeq 2 \frac{D_{A} \tau_{B}}{R_{A B}} \ll R_{A B} .
$$

Result (90) may be rewritten as the ratio of times in view of the fact that the residence time of a diffusing particle in a thin spherical layer of the thickness $\delta$ of the radius $R_{A B}$ is $\delta R_{A B} / D_{A}$ [22]. Really, expression (90) can be recast as

$$
\frac{\left(r_{0}-R_{A B}\right) R_{A B}}{2 D_{A}} \simeq \tau_{B} .
$$

It follows from the above relation (91) that maximum time correlations are realized when the residence time of diffusing $A$ reactant in a thin layer $\left(r_{0}-R_{A B}\right) / 2$ is equal to the time $\tau_{B}$ between $B$ reactant jumps. Note that in this case relative deviations (88) are not as large as they are in the analysis of geminate reaction product accumulation, however, they are proportional to the density parameter, and, therefore, can be measured in the frame of the binary approximation.

Figure 8 shows the dependence of the relative deviation $\Delta_{B}^{s}$ versus parameter $\eta$. Underestimating the rate of geminate reaction, the superposition approximation thus overestimates the probability of $B$ reactant to survive and escape into the bulk. Apparently, $\Delta_{B}^{s}$ is always negative. The largest relative deviations are observed for small $\eta$ when the contribution of geminate reaction to total kinetics $\mathcal{P}_{B}(t)$ is most substantial.

\section{Conclusion}

In this brief review the "scavenger problem" (1), (2) is analyzed with two examples of many-particle model of reacting particles that admits exact analytical solution for any concentrations of bulk acceptors. The derived exact solutions are compared with the results of approximate theories [1-11]

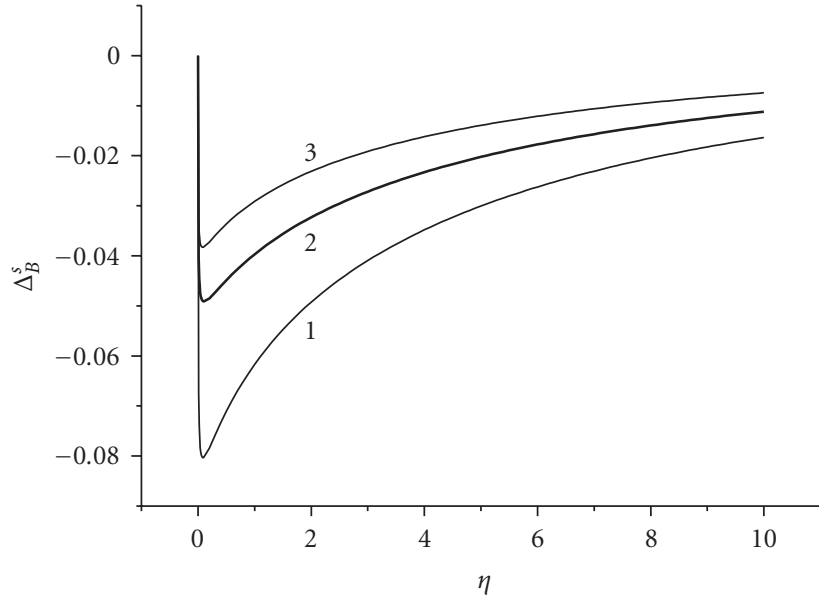

Figure 8: Relative deviation $\Delta_{B}^{s}$ for the diffusing scavengers (2) and its Markovian part (1) versus $\eta=\tilde{\tau}_{g} / \tau_{B}$. Line 3 is the $\Delta_{B}^{s}$ for the model of hopping scavengers. Parameters: $\xi=0.1, \tau_{B} / \tau_{C}=$ $3 \tau_{B} D_{C} / R_{B C}^{2}=0.3$.

widely used to describe experimental data. They are shown to coincide only in the limiting case where the mobility of excess electron (geminate $B$ partner) involved in the competing bulk reaction is equal to zero. In this case time correlations between bulk and geminate reactions are absent at any concentrations of acceptors. Thus the accumulation rate $\dot{\mathcal{P}}_{P}$ of geminate reaction products and the decay kinetics $\mathcal{P}_{B}$ of $B$ reactant are expressed as the product of bulk kinetics (31), (38) and, respectively, geminate reaction rate (22) and geminate kinetics (27):

$$
\dot{\mathcal{P}}_{P}(t)=\tilde{N}(t) \tilde{K}_{g}(t), \quad \mathcal{P}_{B}(t)=\tilde{N}(t) \tilde{\Omega}(t), \quad \frac{1}{\tau_{B}}=0 .
$$

As soon as $B$ reactant starts moving, time correlations appear in the system. As a consequence, the rate in a geminate channel ceases to be proportional to the bulk reaction kinetics, as it was in the case of independent course of reactions (92). When $B$ reactant becomes mobile, the bulk channel is modified accordingly to formulae (49), (59), but the rate in the geminate channel is equal to the product of pure geminate reaction rate $K_{g}(t)(22)$ and the kinetics $\tilde{N}(t)$ formed solely during the residence time of $B$ particle in a geminate pair: $\dot{\mathcal{P}}_{P}(t)=\tilde{N}(t) K_{g}(t)$. As a result, the reaction rate in the geminate channel differs strongly from the prediction following from the superposition approximation: $\dot{\mathcal{P}}_{P}^{s p}(t)=K_{g}(t) N(t)$. This, in turn, leads to the fact that the superposition approximation substantially underestimates the yield of geminate reaction products in the cases where the bulk reaction proceeds deeply during geminate pair lifetime. These distinctions are more pronounced in the case of diffusion motion of acceptors rather than in that of the hopping one.

Another consequence of time correlations between bulk and geminate channels of reactions is that the survival probability $\mathcal{P}_{B}(t)$ of $B$ reactant cannot be represented as a product of the bulk kinetics and the kinetics of geminate 
reaction proceeding independently in some effective pair. If considered in such a way, formula (53) gives rise to a physical contradiction: taking account of additional decay channel in effective acceptor surroundings accelerates the reaction in effective geminate pair, though obviously it can do nothing but slow it down.

Evidently, large distinctions in the theoretical treatment of the measured quantities are to affect experimental data interpretation. In a classical variant [21] the experiment is run as follows: the dependence of the scavenging yield $F$ on the concentration $[C]$ of bulk acceptors is measured with the subsequent determination of the geminate pair kinetics $\Omega(t)$ from the measured concentration dependence $F(\alpha[C])$. Here $\alpha$ is the experimentally measured constant depending on the initial distance distribution in geminate pair and acceptor and solvent properties. Commonly, conventional theory formula (8) is employed assuming that the scavenging yield is the Laplace transform of geminate reaction kinetics:

$$
\mathcal{P}_{D}^{c t}(t \longrightarrow \infty)=[C] k \int_{0}^{\infty} d t \Omega(t) e^{-[C] k t} \approx F(\alpha[C]) .
$$

The inverse Laplace transformation of (93) with $[C] k$ as transformation variable gives the geminate kinetics $\Omega(t)$. Its characteristic decay time is determined from the ratio $\tau_{g} \sim \alpha / k$, with the rate constant value $k$ of the bulk reaction taken from independent experiments on pure bulk reaction. However, it follows from the exact solution (46) that even if "instantaneous quenching" effect is neglected, formula (93) can be reproduced but with an essential distinction: instead of the bulk reaction rate $[C] k$ (equal to $\xi\left(1 / \tau_{C}+1 / \tau_{B}\right.$ ) for hopping and $\xi\left(1 / \tau_{C}+1 / \tau_{B}+\sqrt{3 /\left(\tau_{C} \tau_{B}\right)}\right)$ for diffusing scavengers), the role of the Laplace variable will be played by the reaction rate with immobile $B$ reactant: $[C] \tilde{k}=\xi / \tau_{C}$ that can be essentially less than $[C] k$. Thus the value $\tau_{g}$ found by formula (93) is $k / \tilde{k}$ times less than it actually is. For instance, in liquid hydrocarbons where the mobility of an excess electron substantially exceeds that of acceptors, the constant $k$ is determined mainly by electron mobility. Indeed, using both the lifetime of the excess electron in the localized state in cyclohexane $\tau_{B} \sim 10^{-10} \mathrm{~s}$ [43] and typical values $R_{B C}=10 \AA$ and $D_{C}=10^{-5} \mathrm{~cm}^{2} / \mathrm{s}$, we get $k / \tilde{k} \approx$ 7.5. Note that this value is almost twice greater than the corresponding ratio $k / \tilde{k}$ for the hopping motion of acceptors. Since for our model, according to $(23), \tau_{g} \approx 3 \sqrt{\tilde{\tau}_{g} \tau_{B}}=$ $3\left(r_{0}-R_{A B}\right) \sqrt{\tau_{B} / D_{A}}$, the value of the initial distance in the geminate pair decreases similarly. In real situation when the initial distance in geminate pair $r_{0}$ is not fixed but is distributed according to $P\left(r_{0}\right)$ the characteristic time of geminate reaction is proportional to the width $L$ on the distribution: $\tau_{g} \approx L \sqrt{\tau_{B} / D_{A}}$. So one can considerably underestimate the width of required distribution.

Another part of experimental works is concerned with the rate constant of the bulk reaction of a geminate partner decay on acceptors. In particular, paper [48] suggests an ingenious method of excluding pure geminate kinetics from geminate reaction channel based on formula (14) of the superposition decoupling: $\dot{\mathcal{P}}_{P}^{s p}(t)=K_{g}(t) N(t)$. It is seen from this formula that the bulk kinetics $N(t)$ may be obtained by dividing the experimentally measured fluorescence rate $\dot{\mathcal{P}}_{P}^{s p}(t)$ of geminate reaction products in the presence of acceptors by the fluorescence rate $K_{g}(t)$ of pure geminate reaction products. However, as follows from exact solution (46), the kinetics $\tilde{N}(t)$ thus obtained is not the bulk kinetics and can differ considerably from it. Since the distinction of the exact model and superposition approximation is determined by the difference between the bulk kinetics $N(t)$ and kinetics $\tilde{N}(t)$ the relative deviations $\Delta_{P \text { dot }}$ does not depend of the distribution of initial distances in geminate pair.

In conclusion it should be noted that the exactly solvable model considered above refers to the hopping motion of excess electrons with the mean length of a jump large enough for an electron to leave geminate pair in one jump and change its surroundings in the process of random walks in the bulk in an uncorrelated way. Such a model of excess electron mobility appears to be realizable in such hydrocarbons as isooctane, neopentane, where the mean free path of an excess electron is about $60-200 \AA[23,43,45]$. On the other hand, due to a large value of Onsager radius in such systems $(\sim 300 \AA)$ it is necessary for an electron to execute several jumps to leave geminate pair and escape into the bulk, so an addition correlation caused by Coulomb attraction should be taken into account. That is why, strictly speaking, our many-particle exactly solvable model can first of all be used to describe photodetachment processes of a high mobility excess electron generation in solution.

Also it should be noted that the exactly solvable model was used to establish the form of kinetic equations [60, 61]. It is consistent with the concepts of general kinetic theory [62], according to which in the general case these equations are inhomogeneous integrodifferential ones. This allows one to elucidate the physical meaning of the involved kinetic coefficients, and thus to use the exactly solvable model for testing the kinetic equations obtained on the basis of consistent many-particle approach for a more general character of reactant mobility.

\section{Acknowledgments}

The authors are grateful to the Russian Foundation of Fundamental Research (projects 05-03-32651, 09-03-00456) and to the Program of Fundamental Research of Chemical Division and Material Science RAS (project 5.1.5) for the support of this work.

\section{References}

[1] L. Monchick, "Note on the theory of diffusion controlled reactions: application to photodissociation in solution," The Journal of Chemical Physics, vol. 24, no. 2, pp. 381-385, 1956.

[2] A. Hummel, "Lifetime of charged species in irradiated dielectric liquids," The Journal of Chemical Physics, vol. 49, no. 11, pp. 4840-4844, 1968.

[3] A. Mozumder, "Model for neutralization and scavenging of charges in irradiated dielectric liquids. I. Evolution of the neutralization process in multiple ion-pair cases," The Journal of Chemical Physics, vol. 55, no. 6, pp. 3020-3025, 1971. 
[4] J. L. Magee and A. B. Tayler, "Recombination of ions in a coulomb field in the presence of a scavenger," The Journal of Chemical Physics, vol. 56, no. 6, pp. 3066-3070, 1972.

[5] G. C. Abell, A. Mozumder, and J. L. Magee, "Laplace transform method in the theory of ion neutralization. Application to scavenging probability in the y radiolysis of dielectric liquids," The Journal of Chemical Physics, vol. 56, no. 11, pp. 5422-5427, 1972.

[6] M. Tachiya, "Recombination of Geminate Species in the Presence of a Scavenger ," The Journal of Chemical Physics, vol. 70, p. 238, 1979.

[7] H. Sano and M. Tachiya, "Partially diffusion-controlled recombination," The Journal of Chemical Physics, vol. 71, no. 3, pp. 1276-1282, 1979.

[8] J. A. Crumb and J. K. Balrd, "Reactivity/rate constant ratio for scavengers of charged particles in nonpolar liquids," Journal of Physical Chemistry, vol. 83, no. 9, pp. 1130-1133, 1979.

[9] R. J. Friauf, J. Noolandi, and K. M. Hong, "Calculation of scavenging yield from the initial distribution of electrons in radiolysis of hydrocarbon solutions," The Journal of Chemical Physics, vol. 71, no. 1, pp. 143-147, 1979.

[10] S. Raaen and P. C. Hemmer, "The scavenger problem in diffusion-controlled recombination," The Journal of Chemical Physics, vol. 76, no. 5, pp. 2569-2575, 1982.

[11] J. Kim, Y. Jung, J. Jeon, K. J. Shin, and S. Lee, "Diffusioninfluenced radical recombination in the presence of a scavenger," Journal of Chemical Physics, vol. 104, no. 15, pp. 57845797, 1996.

[12] T. R. Waite, "Diffusion-limited annealing of radiation damage in germanium," Physical Review, vol. 107, no. 2, pp. 471-478, 1957.

[13] N. Kalnin and F. V. Pirogov, "On rate equations in theory of diffusion-controlled reactions," Physica Status Solidi B, vol. 84, no. 2, pp. 521-527, 1977.

[14] W. Naumann, "Interplay between pair and bulk recombination. A stochastic approach to Waite's equation," Chemical Physics, vol. 144, no. 2, pp. 147-153, 1990.

[15] N. Agmon and A. Szabo, "Theory of reversible diffusioninfluenced reactions," The Journal of Chemical Physics, vol. 92, no. 9, pp. 5270-5284, 1990.

[16] J. B. Miers, J. C. Postlewaite, T. Zyung et al., "Diffusion can explain the nonexponential rebinding of carbon monoxide to protoheme," The Journal of Chemical Physics, vol. 93, no. 12, pp. 8771-8776, 1990.

[17] Y. Jung and S. Lee, "Equivalence of the radical recombination rate theories of Waite and Szabo," Chemical Physics Letters, vol. 231, no. 4-6, pp. 429-438, 1994.

[18] T. W. Scott and C. Doubleday Jr., "Picosecond photolysis of azo compounds in liquid alkanes: germinate recombination kinetics for polyatomic free radical pairs," Chemical Physics Letters, vol. 178, no. 1, pp. 9-18, 1991.

[19] M. G. Hyde and G. S. Beddard, "Picosecond photodissociation and geminate radical recombination in alkane solutions of tetraphenylhydrazine," Chemical Physics, vol. 151, no. 2, pp. 239-248, 1991.

[20] L. Onsager, "Initial recombination of ions," Physical Review, vol. 54, no. 8, pp. 554-557, 1938.

[21] S. A. Rice, "Diffuision Limited Reaction," in Comprehensive Chemical Kinetics, C.H. Bamford, C. F. H. Tipper, and R. G. Compton, Eds., vol. 25, Elsevier, Amsterdam, The Netherlands, 1985.

[22] A. A. Ovchinnikov, S. F. Timashev, and A. A. Belyy, Kinetics of Diffusion Controlled Chemical Processes, Nova Science, New York, NY, USA, 1989.
[23] S. Yakovlev, "Excess electrons in non-polar molecular liquids," Russian Chemical Reviews, vol. 48, p. 615, 1979.

[24] A. Hummel, "Single-pair diffusion model of radiolysis of hydrocarbobs," in Kinetics of Nogomogeneous Processes, G. R. Freeman, Ed., John Wiley \& Sons, New York, NY, USA, 1987.

[25] A. Mozumder, Fundamentals of Radiation Chemistry, Academic Press, San Diego, Calif, USA, 1999.

[26] M. Wojcik, M. Tachiya, S. Tagawa, and Y. Hatano, "Electronion recombination in condenced matter: geminate and bulk processes," in Charge Particle and Photon Interactions with Matter, A. Mozumder and Y. Hatano, Eds., Marcel Dekker, New York, NY, USA, 2004.

[27] J. A. Kloepfer, V. H. Vilchiz, V. A. Lenchenkov, X. Chen, and S. E. Bradforth, "Time-resolved scavenging and recombination dynamics from I: e- caged pairs," Journal of Chemical Physics, vol. 117, no. 2, pp. 766-778, 2002.

[28] T. Zhang, Y. J. Lee, T. W. Kee, and P. F. Barbara, "The mechanism of electron-cation geminate recombination in liquid isooctane," Chemical Physics Letters, vol. 403, no. 4-6, pp. 257-261, 2005.

[29] A. T. Healy, D. F. Underwood, S. Lipsky, and D. A. Blank, "Three-photon near-threshold photoionization dynamics of isooctane," Journal of Chemical Physics, vol. 123, no. 5, Article ID 051105, pp. 1-3, 2005.

[30] A. Saeki, T. Kozawa, Y. Ohnishi, and S. Tagawa, "Reactivity between biphenyl and precursor of solvated electrons in tetrahydrofuran measured by picosecond pulse radiolysis in near-ultraviolet, visible, and infrared," Journal of Physical Chemistry A, vol. 111, no. 7, pp. 1229-1235, 2007.

[31] A. Szabo, "Theoretical approaches to reversible diffusioninfluenced reactions: monomer-excimer kinetics," The Journal of Chemical Physics, vol. 95, no. 4, pp. 2481-2490, 1991.

[32] W. Naumann and A. Szabo, "Comparison of the Smoluchowski approach with modern alternative approaches to diffusion-influenced fluorescence quenching: the effect of intense excitation pulses," Journal of Chemical Physics, vol. 107, no. 2, pp. 402-407, 1997.

[33] A. A. Kipriyanov, O. A. Igoshin, and A. B. Doktorov, "New approach to the derivation of binary non-Markovian kinetic equations," Physica A, vol. 268, no. 3-4, pp. 567-606, 1999.

[34] S. G. Fedorenko, A. A. Kipriyanov, and A. B. Doktorov, "Exactly solvable models of a geminate reaction in the presence of scavengers," Physica A, vol. 345, no. 3-4, pp. 421434, 2005.

[35] A. A. Kipriyanov, S. G. Fedorenko, and A. B. Doktorov, "Kinetic equations for exactly solvable models of a geminate reaction in the presence of scavengers," Chemical Physics, vol. 320, no. 1, pp. 9-20, 2005.

[36] M. V. Artamonova, Ch. M. Briskina, A. I. Burshtein, L. D. Zusman, and A. G. Skleznev, "The variation of $\mathrm{Nd}^{3+}$ ion luminescence and an estimation of electron excitation migration along the ions in glass," Soviet Physics-JETP, vol. 35, p. 457, 1972.

[37] A. I. Burshtein, "Jump mechanism of energy transfer," Soviet Physics-JETP, vol. 35, p. 882, 1972.

[38] A. B. Doktorov, A. A. Kipriyanov, and A. I. Burshtein, "Effect of the kinematics approach of particles to each other in solutions on the transfer of energy between them," Soviet Physics-JETP, vol. 47, p. 623, 1978.

[39] A. I. Burshtein, A. B. Doktorov, A. A. Kipriyanov, V.A. Morozov, and S. G. Fedorenko, "Separation of kinematic mechanisms of bimolecular processes," Soviet Physics-JETP, vol. 61, p. 516, 1985. 
[40] A. I. Burshtein and S. G. Fedorenko, "Hopping reactions of charged particles," Journal of Chemical Physics, vol. 106, no. 7, pp. 2662-2669, 1997.

[41] S. G. Fedorenko and A. I. Burshtein, "Electric field suppression of hopping charge recombination," Journal of Chemical Physics, vol. 107, no. 17, pp. 6659-6666, 1997.

[42] S. G. Fedorenko, E. B. Krissinel, and A. I. Burshtein, "Electric field impact on solvated electron reactions: trapping of randomly walking electron," Journal of Chemical Physics, vol. 111, no. 13, pp. 6016-6025, 1999.

[43] B. S. Yakovlev, S. Vash, and A. A. Balakin, "Hopping model of excess electron reactions in liquid hydrocarbons," High Energy Chemistry, vol. 16, p. 139, 1982.

[44] S. G. Fedorenko and A. I. Burshtein, "Tunneling of excess electron from free and trapped states," Journal of Chemical Physics, vol. 121, no. 23, pp. 11876-11884, 2004.

[45] K. Shinsaka and Y. Hatano, "Electron-ion recombination in condensed nonpolar media," Nuclear Instruments and Methods in Physics Research, A, vol. 327, no. 1, pp. 7-10, 1993.

[46] S. G. Fedorenko and A. A. Kipriyanov, "Geminate recombination in the presence of scavengers: breakdown of the superposition approximation," Chemical Physics Letters, vol. 428, no. 1-3, pp. 200-206, 2006.

[47] S. G. Fedorenko, A. A. Kipriyanov, and A. B. Doktorov, "Recombination and scavenging of a randomly walking excess electron in solution," Chemical Physics Letters, vol. 436, no. 13, pp. 104-110, 2007.

[48] V. I. Borovkov and K. A. Velizhanin, "A novel approach to study radical ion reactions in the course of geminate recombination by the quenching of time-resolved delayed fluorescence," Chemical Physics Letters, vol. 394, no. 4-6, pp. 441-445, 2004.

[49] N. J. B. Green and S. M. Pimblott, "Scavenger kinetics AND the laplace transform relationship.2 nonhomogeneous," Molecular Physics, vol. 74, pp. 811-832, 1991.

[50] N. J. B. Green, S. M. Pimblott, and M. Tachiya, "Generalizations of the Stern-Volmer relation," Journal of Physical Chemistry, vol. 97, no. 1, pp. 196-202, 1993.

[51] I. Z. Steinberg and E. Katchalski, "Theoretical analysis of the role of diffusion in chemical reactions, fluorescence quenching, and nonradiative energy transfer," The Journal of Chemical Physics, vol. 48, no. 6, pp. 2404-2410, 1968.

[52] M. Tachiya, "Theory of diffusion-controlled reactions: formulation of the bulk reaction rate in terms of the pair probability," Radiation Physics and Chemistry, vol. 21, no. 1-2, pp. 167-175, 1983.

[53] M. Smoluchowski, "Versuch einer mathematischen Theorie der Koagulationskinetik kolloider Lösungen," Zeitschrift für Physikalische Chemie, vol. 92, pp. 129-168, 1917.

[54] A. A. Kipriyanov and A. A. Karpushin, "Two-scale migration in theory of excitation transfer in condence matter," Khimicheskaya Fizika, vol. 7, p. 60, 1988.

[55] P. Clifford, N. J. B. Green, and M. J. Pilling, "Analysis of the Debye-Smoluchowski equation. Approximations for highpermittivity solvents," Journal of Physical Chemistry, vol. 88, no. 18, pp. 4171-4176, 1984.

[56] N. J.B. Green, "Reaction probability and diffusion-controlled rate constants for ionic reactions in solvents of high permittivity," Chemical Physics Letters, vol. 107, no. 4-5, pp. 485-488, 1984.

[57] I. M. Frank and S. I. Wawilow, "Über die Wirkungssphäre der Auslöschungsvorgänge in den fluoreszierenden Flüssigkeiten," Zeitschrift für Physik, vol. 69, no. 1-2, pp. 100-110, 1931.
[58] A. A. Kipriyanov, I. V. Gopich, and A. B. Doktorov, "A modification of non-Markovian encounter theory. I. Markovian description in non-Markovian theories," Chemical Physics, vol. 187, no. 3, pp. 241-249, 1994.

[59] M. Abramowitz and I. I. Stegun, Eds., Handbook of Mathematical Functions, Dover, New York, NY, USA, 1965.

[60] A. A. Kipriyanov, S. G. Fedorenko, and A. B. Doktorov, "Kinetic equations for exactly solvable models of a geminate reaction in the presence of scavengers," Chemical Physics, vol. 320, no. 1, pp. 9-20, 2005.

[61] A. A. Kipriyanov and A. B. Doktorov, "Kinetic equations for exactly solvable models of geminate reactions in the presence of scavengers," Khimicheskaya Fizika, vol. 26, no. 2, pp. 36-45, 2007 (Russian).

[62] R. Balescu, Equilibrium and Nonequilibrium Statistical Mechanics, vol. 2, John Wiley \& Sons, New York, NY, USA, 1978. 

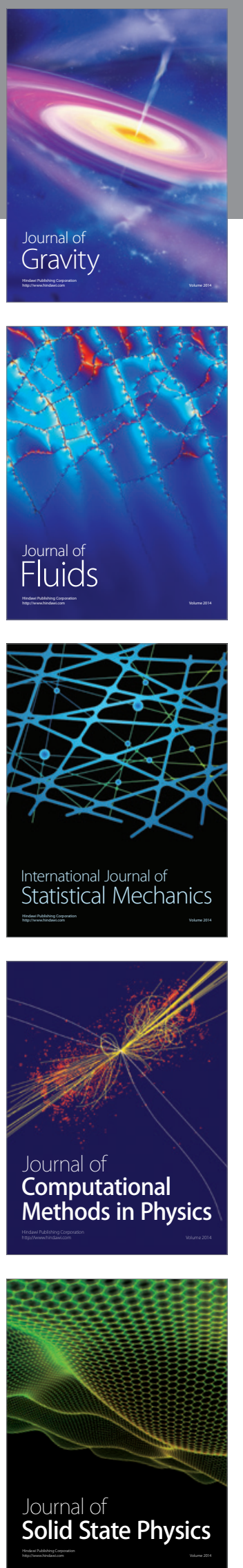

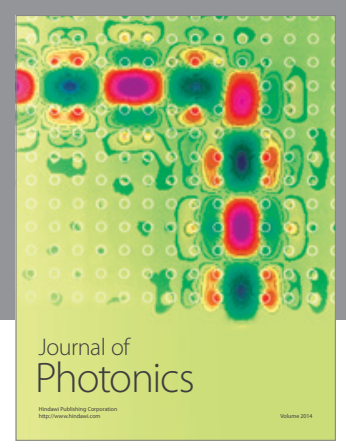

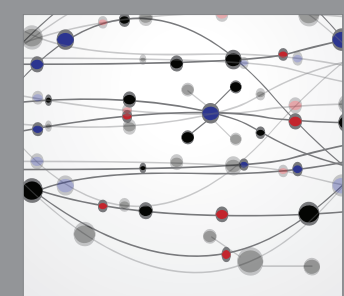

The Scientific World Journal
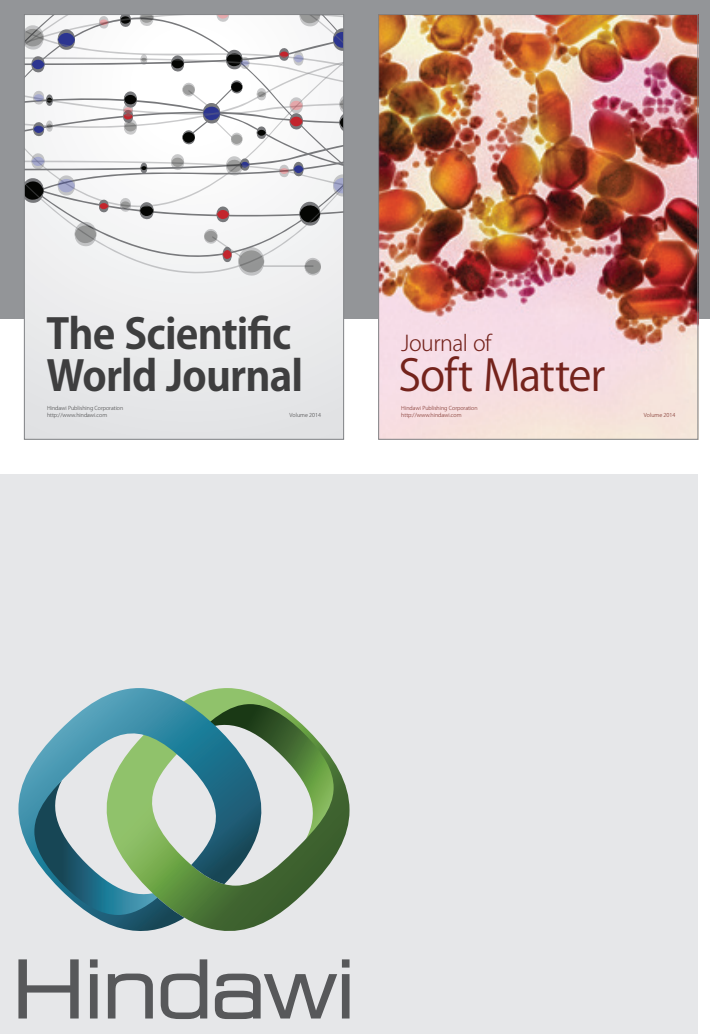

Submit your manuscripts at

http://www.hindawi.com
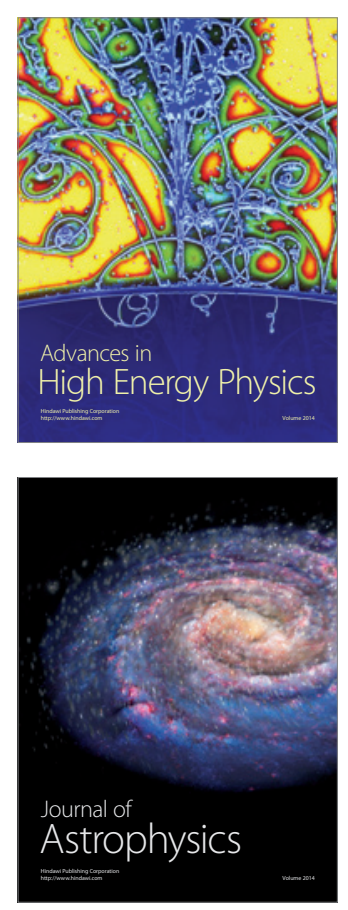
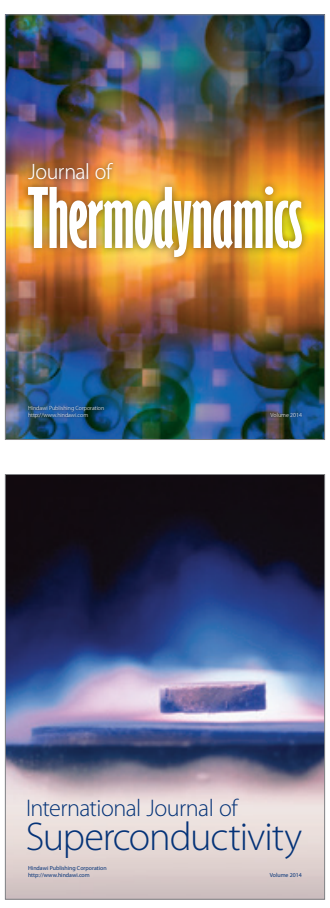
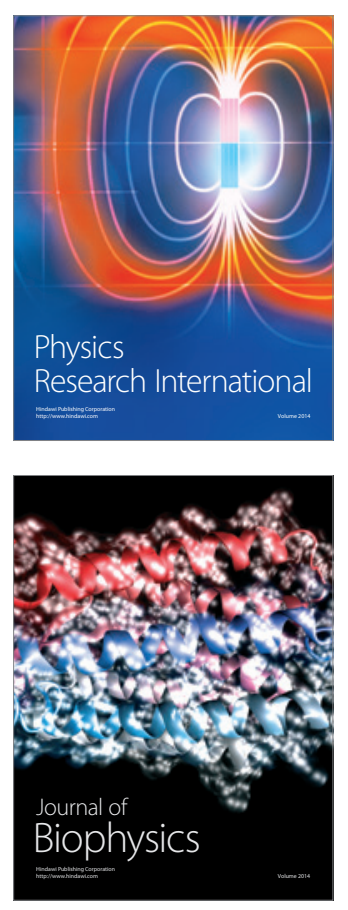
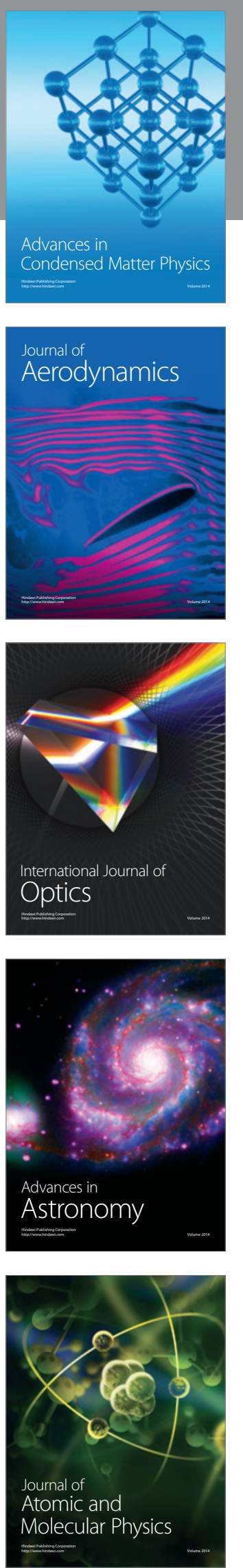